\title{
RNA degradation sculpts the maternal transcriptome during Drosophila oogenesis
}

\author{
Patrick Blatt ${ }^{1}$, Siu Wah Wong-Deyrup ${ }^{1}$, Alicia McCarthy ${ }^{1,2}$, Shane Breznak ${ }^{1}$, Matthew D. Hurton ${ }^{3}$, \\ Maitreyi Upadhyay ${ }^{1,4}$, Benjamin Bennink ${ }^{1}$, Justin Camacho ${ }^{1}$, Miler T. Lee ${ }^{* 3}$, Prashanth Rangan ${ }^{* 1}$ \\ ${ }^{1}$ University at Albany, Department of Biological Sciences, RNA Institute; 1400 Washington \\ Avenue, LSRB 2033D, Albany, NY 12222 \\ ${ }^{2} 10 x$ Genomics, Inc., 6230 Stoneridge Mall Road, Pleasanton, CA, 94588 \\ ${ }^{3}$ University of Pittsburgh, Department of Biological Sciences; 4249 Fifth Avenue, Pittsburgh, PA \\ 15260 \\ ${ }^{4}$ Department of Stem Cell and Regenerative Biology, Sherman Fairchild 100, Harvard \\ University, 7 Divinity Avenue, Cambridge, MA 02138 \\ *Co-Corresponding authors: miler@pitt.edu \& prangan@albany.edu
}

\begin{abstract}
In sexually reproducing animals, the oocyte contributes a large supply of RNAs that are essential to launch development upon fertilization. The mechanisms that regulate the composition of the maternal RNA contribution during oogenesis are unclear. Here, we show that a subset of RNAs expressed during the early stages of oogenesis is subjected to regulated degradation during oocyte specification. Failure to remove these RNAs results in oocyte dysfunction and death. We identify the RNA-degrading Super Killer complex and No-Go Decay factor Pelota as key regulators of oogenesis via targeted clearance of RNAs expressed in germline stem cells. These regulators target RNAs enriched for cytidine sequences bound by the protein Half pint. Thus, RNA degradation helps orchestrate a germ cell-to-maternal transition by sculpting the maternal RNA contribution to the zygote.
\end{abstract}

\section{Report}

A fertilized egg is totipotent, having the unique potential to differentiate into every cell lineage in the adult organism ${ }^{1-3}$. Across animals, $40-75 \%$ of genes are deposited into the egg during oogenesis as part of the maternal RNA contribution required for embryo development ${ }^{4-6}$. It is unlikely that every RNA synthesized during oogenesis is destined for the maternal contribution: RNAs that support oogenesis-specific functions, such as germline stem cell (GSC) self-renewal and differentiation, could be detrimental during embryogenesis. It is not known if such oogenesisspecific RNAs are targeted for elimination or what, if any, mechanisms ensure that only the appropriate RNAs are deposited into the oocyte.

In Drosophila, oogenesis occurs in ovarioles composed of germaria, which contain the GSCs and the GSC daughter cells (cystoblasts, CB) that progressively differentiate into 16-cell cysts (Figure $1 A)^{7-11}$. In each cyst, the oocyte receives RNA and protein contributions from the remaining 15 nurse cells (Figure $1 A^{\prime}$ ), thus causing the oocyte to enlarge forming egg chambers (Figure $\left.1 \mathrm{~A}\right)^{12-}$ 18. In a screen to identify novel regulators of this process, we discovered that a component of the RNA-degradation-promoting Super Killer (Ski) complex (Figure 1B), Super Killer 2 (Ski2), called Twister (Tst) in Drosophila, is required for egg chamber growth and female fertility (Figure S1A) ${ }^{19-}$ 22. Wild type (WT) Drosophila ovarioles stained for Vasa (germ cells) and for 1B1 (somatic cell membranes) show the progression from the germarium to successively larger egg chambers (Figure 1C). In contrast, egg chambers failed to grow in tst mutant ovarioles (Figure 1C-D, 1M) as well as upon germline RNAi depletion of tst (nanos-GAL4 >RNAi, Figure 1E-F, 1M) but not when tst was depleted in the soma (traffic jam-GAL4 >RNAi, S1B-C) ${ }^{23,24}$. However, tst mutant flies are otherwise viable, and successful oogenesis and egg production were restored in tst mutants by expressing Tst protein in the germline alone (Figure 1G-H, 1M, S1A). Egg chambers 
lacking tst expressed cleaved Caspase 3 at putative stages 6-7, suggesting that they undergo apoptosis (Figure S1D-E).

The Ski complex, in addition to RNA helicase SKI2, consists of the scaffolding subunits SKI3 and SKI8, which are coupled to the exosome complex by SKI7 (Figure 1B) $20,21,25,26$. We found that ski3 (CG8777) mutant and germline depletion of ski3 and ski8 (CG3909) phenocopied tst mutants (Figure 1I-M, S1F-H). HBS1 is thought to fulfill the role of SKI7 in Drosophila; however, female hbs 1 mutants were previously found to be fertile, suggesting that SKI7/HBS1 is dispensable for Ski complex function in the female germline or acts redundantly with a yet-unidentified protein ${ }^{27-}$ ${ }^{29}$. Overall, we conclude that the Ski complex components Ski2, Ski3 and Ski8 are required in the fly germ line for oogenesis.

Given the role of the Ski complex in exosome-mediated RNA degradation, we hypothesized that Tst promotes degradation of RNAs during oogenesis ${ }^{21,30,31}$. RNA sequencing (RNA-seq) revealed 296 genes upregulated in ovaries lacking tst (Figure 2A, Supplemental Table 1). These include 207 genes such as blanks and actin 57B (act57B) with >4-fold higher levels in a germline tst RNAi compared to WT (Figure 2B), which likely represent transcripts regulated by Tst in the germline. To determine that the depletion of tst resulted in a defect in post-transcriptional regulation, we measured pre-mRNA levels of select Tst-regulated RNAs by qRT-PCR and indeed found no significant difference between WT and tst germline RNAi flies (Figure S2A). Taken together, these data suggest that $t s t$ promotes the post-transcriptional degradation of a distinct group of RNAs during oogenesis.

To determine when Tst acts, we used RNA-seq to profile the expression of Tst-regulated RNAs in ovaries across a time course of oocyte development: GSCs, CBs, and cysts, which were each enriched using mutants (see Methods); germaria and early egg chambers were enriched using young WT ovaries; late-stage egg chambers were enriched using adult WT ovaries; and unfertilized eggs, which represent the maternal contribution ${ }^{9,11,32-34}$. Principal component analysis revealed that tst mutant and tst RNAi ovaries more closely resemble WT as compared to undifferentiated stages, suggesting that tst is required after differentiation (Figure S2B). Indeed, compared to non-targets, Tst-regulated RNAs decreased at the cyst stages and were nearly absent as part of the maternal contribution in the egg (Figure 2C, S2C-D). In situ hybridization of the Tst-regulated RNAs blanks and act57B demonstrates low levels beginning in the cyst stages in WT, in contrast to persistence throughout the egg chambers in tst germline RNAi (Figure 2DG'). To precisely determine when Tst-regulated RNAs are degraded, we probed for proteins encoded by Tst-regulated RNAs blanks and actins. In WT, both Blanks and nuclear-Actins (detected by C4 staining) were highly expressed in GSCs and CBs but their expression is attenuated in the cysts, when the oocyte is specified, consistent with previous reports (Figure $2 \mathrm{H}$ $M)^{35-38}$. In contrast, both Blanks and nuclear-Actin expression persisted in the cysts and egg chambers of tst germline RNAi flies (Figure $2 \mathrm{H}-\mathrm{M}$ ). We did not find gross changes to cytoplasmic Actin pool upon the loss of $t s t$, as measured by Phalloidin staining (Figure S2E-F') ${ }^{39}$. Overall, our data suggest that Tst attenuates the levels of Blanks and Actin proteins by degrading their mRNAs before oocyte specification.

To investigate how specific transcripts are targeted by Tst, we considered the contribution of RNA surveillance pathways, which are known to direct RNAs to the Ski complex for degradation ${ }^{40}$. Nonsense mediated decay (NMD) and non-stop decay (NSD) are unlikely to be involved. In contrast to tst, ski3 and ski8 germline RNAi flies, germline mutant clones of the NMD pathway components up-frameshift 1 (Upf1), Upf2, and Upf3 do produce eggs, albeit with patterning 100 defects ${ }^{41}$. We additionally looked for features in the RNAs that could trigger NMD or NSD. Most 
102

103

104

105

106

107

108

109

110

111

112

113

114

115

116

117

118

119

120

121

122

123

124

125

126

127

128

129

130

131

132

133

134

135

136

137

138

139

140

141

142

143

144

145

146

147

148

149

150

151

nor show any evidence for aberrant splicing that would give rise to premature termination codons (Figure S3B), ruling out NMD ${ }^{42-44}$. NSD is triggered by ribosome read through into the 3' UTR, but all Tst-regulated RNAs are annotated transcripts that encode stop codons suggesting that NSD is also not involved ${ }^{45-47}$.

However, we did find evidence that no-go decay (NGD), which is activated when ribosomes stall on RNAs, was involved in the degradation of Tst-regulated RNAs. Pelota (Pelo/DOM34) is a critical effector protein of the NGD pathway that promotes recycling of stalled ribosomes on mRNAs ${ }^{27,48,49}$. Intriguingly, pelo mutants, like tst mutants, are homozygous viable but female sterile, and this role is germline specific ${ }^{50}$. pelo mutant egg chambers failed to grow and died midoogenesis, phenocopying tst mutant ovaries (Figure $3 \mathrm{~A}-\mathrm{C}$ ). In addition, pelo mutants also lost GSCs, as previously described (Figure S3C-D) ${ }^{50}$. To test if pelo and tst co-regulate target RNAs, we performed RNA-seq on pelo mutant ovaries and found that $81 \%$ of genes upregulated upon the loss of $t s t$ were also upregulated $>2$-fold in pelo mutants, including act57B, though not blanks (168/207, Figure 3D). These data suggest that pelo, a key component of the NGD pathway, promotes the degradation of a large fraction of Tst-regulated RNAs.

To observe the translation dynamics of Tst-regulated RNAs, we purified polysomes from ovaries enriched for different stages of oocyte development and performed RNA-seq (Figure 3E-G). Adult WT ovaries overall show proportional RNA-seq read depth in the polysome fraction (y-axis) compared to total RNA (x-axis), with Tst-regulated RNAs recapitulating the low expression we observed previously (Figure 3E). In undifferentiated CBs, where Tst-regulated RNA levels are higher, we observed weak polysome association (Figure 3F, J), but overall these RNAs appear to be translated; this is consistent with detection of Blanks protein in CBs prior to oocyte specification (Figure $2 \mathrm{H}-\mathrm{H}^{\prime}$ ). In the differentiating cysts, polysome association appears to increase (Figure 3G, J); however, we did not observe Blanks protein in WT cysts and egg chambers (Figure $2 \mathrm{H}-\mathrm{H}^{\prime}$ ), suggesting that ribosome engagement of these Tst-regulated RNAs is not productive. Although increased association with ribosomes is usually linked to increased RNA stability, Tstregulated RNAs showed an increased association with ribosomes concomitant with their degradation (Figure 3E-G, J) ${ }^{51}$. This change in polysome association is not seen for non-targets (Figure S3E). These results suggest ribosomes are stalled on Tst-regulated RNAs prior to their degradation.

As DOM34 (pelo) promotes recycling of stalled ribosomes, and SKI2 is both required to extract RNAs from stalled ribosomes and to promote their degradation, we predicted that Tst-regulated RNAs would be associated with polysomes but not degraded in the later stages of oogenesis in tst and pelo mutants ${ }^{52-54}$. Indeed, we found that Tst-regulated RNA abundance was substantially increased in tst and pelo mutant ovaries compared to developmentally similar WT ovaries, and that these RNAs are associated with ribosomes (Figure 3H-J). Taken together, we find that Tstregulated RNAs have increased association with ribosomes prior to their degradation and are regulated by the NGD pathway member pelo.

Pelo-mediated degradation can be activated by features in the coding sequence that cause ribosome stalling, such as sub-optimal codons ${ }^{55,56}$. We found that Tst-regulated RNAs in fact have an elevated codon optimality compared to non-targets, as measured by the Codon Adaptation Index (CAI), suggesting sub-optimal codon frequency does not trigger degradation of Tst-regulated RNAs (Figure S4A) ${ }^{57,58}$. Instead, we found an enrichment of repeating, interspaced cytidine residues in the coding sequence (CDS), but not in the 5'UTRs or 3'UTRs, of Tst-regulated RNAs (Figure 4A), suggesting cytidine tracts might recruit Tst. To investigate this hypothesis, we compared three actin paralogs (act42A, act57B and act87E), which were upregulated upon the 
152

153

154

155

156

157

158

159

160

161

162

163

164

165

166

167

168

169

170

171

172

173

174

175

176

177

178

179

180

181

182

183

184

185

186

187

188

189

190

191

192

193

194

195

196

197

198

199

200

201

202

loss of $t s t$, to a fourth, act5C, which was not upregulated. The actin coding sequences are highly similar (>84\% nucleotide identity) and have similar CAls (Fig S4B). However, a multiple sequence alignment revealed a repeating cytidine tract in the codon wobble position of act42A, act57B and act87E that is interrupted by purines in the non Tst-target act5C (Figure 4B).

To evaluate the effect of these sequence differences in vivo, we built reporters with the GFP open reading frame fused to the CDS of non-target act5C and target act57B, as well as a version of act57B with cytidine tracts mutated to match act5C (PT-mutant). We expressed these reporters under the control of the maternal germline promoter pgc, as well as the 3'UTR of nos, and 5'UTR of $\mathrm{K} 10$ (Figure S4C-C"), which are not translationally repressed ${ }^{32,59-61}$. Levels of act5C-GFP did not significantly change during oocyte specification or upon loss of tst (Figure 4C-D', H). In contrast, the levels of act57B-GFP were significantly reduced in WT cysts compared to undifferentiated cells (Figure 4E-E', H); we note that the reporter was re-expressed in the egg chambers, which could arise from the strong maternal germline promoter or additional layers of control on Tst-regulated RNAs. Strikingly, upon germline depletion of tst, the levels of act57BGFP were strongly elevated in cysts (Figure 4E-F', H). Expression of act57B PT Mutant-GFP was significantly higher in cysts compared to that of act57B-GFP (Figure 4G-H), matching act5C-GFP and demonstrating the importance of the cytidine tract in promoting destabilization of Tstregulated RNAs during the cyst stages.

To identify the factor that recruits Tst to these cytidine tracts, we looked for polypyrimidine tract binding proteins (PTBs) expressed during oogenesis, and found two: hephaetus (heph) and half pint ( $h f p$ ), the homolog of human PUF60. While loss of heph did not phenocopy pelo and tst, loss of $h f p$ partially phenocopied the oogenesis defects of pelo and tst (Figure $4 \mathrm{I}-\mathrm{K})^{62,63}$. Consistent with previous reports, we found that $\mathrm{Hfp}$ is present in the nucleus, where it has been shown to regulate splicing and we also observed it in the cytoplasm, suggesting it can affect RNA stability as well as translation (Figure S4D-E) ${ }^{63}$. To determine if Hfp preferentially binds to the cytidine tracts found in Tst-regulated RNAs, we performed Electrophoretic Mobility Shift Assays (EMSA) using a recombinant protein composed of the two N-terminal RNA Recognition Motifs (RRM) of $\mathrm{Hfp}$, which dimerize on a denaturing gel (Figure S4F-G). We observed that the Hfp RRMs bound the act57B PT more efficiently than the act5C PT sequence (Figure 4L). To determine if Hfp bound to Tst-regulated RNAs in vivo, we immunoprecipitated Hfp from young WT ovaries followed by qRT-PCR. We found that the Tst-regulated RNA act57B was robustly associated with Hfp, whereas the non-targets polar granule component (pgc) and act5C were not (Figure $\mathrm{S} 4 \mathrm{H})$. Lastly, to determine if Hfp also co-regulates Tst-regulated RNAs, we performed RNA-seq of $h f p$ mutant ovaries. We found that $70 \%$ of the RNAs upregulated in tst-depleted ovaries were also upregulated $>2$-fold in $h f p$ mutants, including act57B, act42A and act87E (144/207, Figure 4M), whereas act5C was not upregulated in either mutant. We did not observe splicing defects of Tstregulated RNAs in $h f p$ mutants, ruling out mis-splicing as the reason for their upregulation (Figure S4I). Taken together, our data suggest that Hfp binding to a subset of Tst-regulated RNAs can elicit their degradation mediated by both Pelo and Tst by presumably modulating ribosome association.

Having elucidated the mechanisms underlying how Tst-regulated RNAs are recognized for degradation, we sought to determine how correct temporal regulation of these RNAs contributes to oogenesis. We first assessed the functions of Tst-regulated RNAs prior to their degradation. Individual depletion of 6 out of 50 Tst-regulated RNAs we tested using germline-specific RNAi resulted in germline defects, including a complete loss of the germline (Supplemental Table 2) (Figure S5A-E). Finally, to elucidate why ectopic persistence of Tst-regulated RNAs interferes with later oogenesis, we examined tst mutants for hallmarks of oogenesis defects. We did not find any changes in differentiation or nurse-cell endocycling (Figure S6A-F) $)^{11,17,64-67}$. However, we did 
203

204

205

206

207

208

209

210

211

212

213

214

215

216

217

218

219

220

221

222

223

224

225

226

227

228

229

230

231

232

233

234

235

236

237

238

239

240

241

242

243

244

245

246

247

248

249

250

251

252

observe that Egalitarian (Egl), a protein required to transport the maternal RNA contribution to the oocyte, always localized to the oocyte in WT but in tst, pelo and hfp mutants, while Egl initially localized to the oocyte, this localization was not maintained in later egg chambers (Figure S6G$\left.J^{\prime}\right)^{13,68}$. This suggests that targeted RNA degradation is required for proper inheritance of the maternal contribution, which is necessary for oocyte specification. Thus, some Tst-regulated RNAs play critical roles in germ stem cell maintenance, but are detrimental to the transition to a mature oocyte.

In conclusion, we find that specific RNAs expressed during the undifferentiated stages of oogenesis are degraded during oocyte specification preventing them from being inherited as part of the maternal contribution mediated by the NGD components Pelo and Tst (Figure 4N). Aberrant persistence of these RNAs results in loss of oocyte maintenance and death of egg chambers. This suggests that precise curation of the maternal contribution is tightly coupled to successful egg production. Based on our observations, we propose that a germ cell-to-maternal transition (GMT) occurs during oocyte specification. We speculate that the GMT exists to enable both the transition from germ cell to oocyte identity and the accrual of the maternal RNA contribution to the embryo. After fertilization, the maternal contribution is subsequently cleared during the oocyteto-embryo and maternal-to-zygotic transitions (MZT) to promote a zygotic identity ${ }^{69-71}$. Thus, RNA degradation bookends an oocyte's fate, regulating both its initiation and termination.

\section{Materials and Methods:}

\section{Fly lines}

Flies were grown at $25^{\circ} \mathrm{C}$ and dissected between 1-3 days post-eclosion. The following RNAi stocks were used in this study: tst RNAi (Bloomington \#55647), CG8777 RNAi (Ski3, VDRC \#v100948), CG3909 RNAi (Ski8, VDRC \#12758), bam RNAi (Bloomington \#33631), UAS-TkV (Bloomington \#35653), bam RNAi; hs-bam ${ }^{33}$. The following tissue-specific drivers were used in this study: UAS-Dcr2;nosGAL4 (Bloomington \#25751), UAS-Dcr2;nosGAL4; bamGFP, If/CyO;nosGAL4 (Lehmann Lab) nosGAL4;MKRS/TM6 (Bloomington \#4442), and tjGAL4/CyO (Lehmann Lab) ${ }^{23}$. The following stocks were used in this study: $y^{1} w^{1118} P\{r y[+t 7.2]=70 F L P\} 3 F$ (Bloomington \#6420), w'1118;Mi\{ET1\}tst ${ }^{M B 10212} / T M 6 C, S b 1$ (Bloomington \#29100), hfp ${ }^{9}, c u / T M 2$ (Schüpbach Lab), M\{UAS-hfp.ORF.3xHA\}ZH-86Fb (FlyORF, F000989), CG8777M102824/CyO (Bloomington \#35904), $\quad w[1118]$;Df(2R)ED1770, $P\{w[+m W . S c e r l F R T . h s 3]=3$ '.RS5+3.3'\} ED1770/SM6a (Bloomington \#9157), w[1118]; Df(3R)Exel9013/TM6B, Tb[1] (Bloomington \#7991), pelo $1 /$ CyO (Bloomington \#11757).

\section{Genotypes used to enrich specific stages of germline:}

Germline Stem Cells: nosGAL4>UAS-Tkv (Bloomington \#35653) nosGAL4>bam RNAi (Bloomington \#33631) ${ }^{11,64,65}$. Differentiating Cysts: nosGAL4>bam RNAi; $h s-b_{a m}{ }^{33}$. Female flies were heat shocked at $37^{\circ} \mathrm{C}$ for 2 hours, incubated at room temperature for 4 hours and heat shocked again for 2 hours. This was subsequently repeated the next day and flies were dissected. Young Wild Type: $y^{1} w^{1118} P\{r y[+t 7.2]=70 F L P\} 3 F$ (Bloomington \#6420). Female flies were collected and dissected within 2 hours of eclosion.

\section{Dissection and Immunostaining}

Flies were dissected in 1X PBS and samples were fixed for 10 minutes in $5 \%$ methanol-free formaldehyde ${ }^{32}$. Ovary samples were washed in $1 \mathrm{~mL}$ PBT (1X PBS, 0.5\% Triton X-100, 0.3\% BSA) 4 times for 7 minutes each. Primary antibodies were added in PBT and incubated at $4^{\circ} \mathrm{C}$ rotating overnight. Samples were washed 4 times for 7 minutes each in $1 \mathrm{~mL}$ PBT, and once in 1 $\mathrm{mL}$ PBT with $2 \%$ donkey serum (Sigma) for 15 minutes. Secondary antibodies were added in PBT 
with $4 \%$ donkey serum and incubated at room temperature for 2 hours. Samples were washed 4 times for 7 minutes each in $1 \mathrm{~mL}$ of $1 \mathrm{X}$ PBST (0.2\% Tween 20 in 1x PBS) and incubated in Vectashield with DAPI (Vector Laboratories) for 30 minutes before mounting. The following primary antibodies were used: Mouse anti-1B1 (1:20, DSHB), Rabbit anti-Vasa (1:1000, Rangan Lab), Chicken anti-Vasa (1:1000), Rabbit anti-GFP (1:2000, Abcam, ab6556), Rabbit anti-Blanks (1:1000, Sontheimer Lab), Mouse anti-Actin C4 (Sigma, MAB1501), Rabbit anti-Cleaved Caspase3 (1:300, Cell Signaling \#96615), Rabbit anti-Egl (1:1000, Lehmann Lab), Alexa 488Conjugated Phalloidin (Cell Signaling \#8878), Mouse anti-Hfp (1:25, Schüpbach Lab) ${ }^{35,37,73,74}$. The following secondary antibodies were used: Alexa 488 (Molecular Probes), Cy3, and Cy5 (Jackson Labs) were used at a dilution of 1:500.

\section{Fluorescence Imaging}

The ovary tissue samples were visualized under 10X dry, 20X dry and 40X oil objective lenses and images were acquired using a Zeiss LSM-710 confocal microscope. Confocal images were processed with ImageJ. A.U. The images were quantified using ImageJ with the Measurement function.

\section{Generation of Transgenic Flies}

The pCasper2 plasmid containing the pgc promoter, nos 5'UTR, eGFP CDS and K10 3'UTR was used as a backbone to generate Actin-GFP reporter constructs ${ }^{32}$. gBlocks (IDT) of the actin5C, actin57B and actin57B PT-Mutant CDSs were individually cloned upstream of GFP by digesting with Spel (NEB, R0133S). Constructs were ligated through Gibson Assembly (NEB, E2611S), utilizing complementary overhangs between the CDS fragment and the pCasper2 backbone. Injection of these plasmids into Drosophila embryos was conducted by BestGene Inc.

\section{Gateway Cloning}

The coding sequence of Tst was PCR amplified from cDNA to include flanking attB sites. BP recombination was carried out according to the manufacturer's protocol using equimolar amounts $(100 \mathrm{fmol})$ of the attB-PCR product and the pDONR entry clone plasmid (Invitrogen, 12535-019). Components were incubated in TE buffer with BP Clonase enzyme mix and reaction buffer at $25^{\circ} \mathrm{C}$ for one hour. $2 \mu \mathrm{g} / \mu \mathrm{L}$ Proteinase $\mathrm{K}$ was added to the reaction and incubated $a 7^{\circ} \mathrm{C}$ for one hour. Plasmid was then transformed into DH5a competent cells and plated on LB-Kan plates at $37^{\circ} \mathrm{C}$ overnight (Thermo, 18265017). Cells of individual colony samples were propagated and plasmid was purified. LR recombination reaction was performed with the pPPW and pPGW destination vectors (Gateway Collection). Components were incubated in TE buffer with LR Clonase enzyme mix and reaction buffer at $25^{\circ} \mathrm{C}$ for one hour. $2 \mu \mathrm{g} / \mu \mathrm{L}$ Proteinase $\mathrm{K}$ was added to the reaction and incubated at $37^{\circ} \mathrm{C}$ for one hour. Plasmid was then transformed into XL10-Gold competent cells and plated on LB-Kan plates at $37^{\circ} \mathrm{C}$ overnight (Integrated Sciences, 200315). Cells of individual colony samples were propagated, plasmid was purified and sequenced to verify insertion.

\section{RNA Isolation}

Ovaries were dissected in 1X PBS and homogenized in 50uL of TRIzol (Invitrogen, 15596026) ${ }^{32}$. RNA was isolated by adding an additional $950 \mathrm{uL}$ of TRIzol and $230 \mathrm{uL}$ of Chloroform with mixing. Samples were centrifuged at $13,000 \mathrm{rpm}, 4^{\circ} \mathrm{C}$ for 15 minutes. Aqueous phase was transferred to a new tube, nucleic acids were precipitated using $1 \mathrm{~mL}$ of $100 \%$ ethanol, $52 \mu \mathrm{L}$ of $3 \mathrm{M}$ Sodium Acetate and precipitated for $>1$ hour at $-20^{\circ} \mathrm{C}$. Samples were centrifuged at $13,000 \mathrm{rpm}, 4^{\circ} \mathrm{C}$ for 20 minutes. Ethanol was decanted, pellet was washed with $70 \%$ ethanol and dried at room temperature for 10 minutes. Pellet was dissolved in $20 \mu \mathrm{L}$ RNase free water and placed in a $42^{\circ} \mathrm{C}$ water bath for 10 minutes. Concentration of nucleic acid samples were measured on a spectrophotometer and treated with DNase (TURBO DNA-free Kit, Life Technologies, AM1907). 
304

\section{Quantitative Real Time-PCR (qRT-PCR)}

$1 \mu \mathrm{L}$ of cDNA was amplified using $5 \mu \mathrm{L}$ of SYBR green Master Mix, $0.3 \mu \mathrm{L}$ of $10 \mu \mathrm{M}$ of each reverse and forward primers in a $10 \mu \mathrm{L}$ reaction ${ }^{32}$. The thermal cycling conditions consisted of $50^{\circ} \mathrm{C}$ for 2 minutes, $95^{\circ} \mathrm{C}$ for 10 minutes, 40 cycles at $95^{\circ} \mathrm{C}$ for 15 seconds, and $60^{\circ} \mathrm{C}$ for 60 seconds. The experiments were carried out in technical triplicate and minimum 2 biological replicates for each sample. rp49 gene was utilized as a control. To calculate fold change in mRNA levels to rp49 mRNA levels, average of the $2^{\wedge} \Delta \mathrm{Ct}$ for the biological replicates was calculated. Error bars were plotted using standard error of the ratios. P-value was determined by Students t-test.

\section{RNAseq library preparation}

Total RNA samples were run on a $1 \%$ agarose gel to assess sample integrity ${ }^{75}$. To generate mRNA-Seq libraries, total RNA was incubated with poly $(A)$ selection beads. mRNA enriched sequencing libraries were made with the NEXTflex Rapid Directional RNAseq Kit (BioO Scientific Corp.) and corresponding protocol. mRNA was fragmented at $95^{\circ} \mathrm{C}$ for 13 minutes to achieve $\sim 300$ bp fragments. $75 \mathrm{bp}$ single-end (or paired-end as specified) mRNA sequencing was performed each sample with an Illumina NextSeq500, carried out by the Center for Functional Genomics (CFG).

\section{RNA-seq analysis}

Sequenced reads were aligned to the $D$. melanogaster genome (UCSCdm6 and FlyBase R6.01) using HISAT2 v2.0.576. Unambiguously mapping reads RefSeq annotated mRNA and lincRNA were quantified using featureCounts v1.5.1 default parameters ${ }^{77}$. Genes with $>=0.5$ reads per million (RPM) in one of WT ovaries, tst mutant ovaries, tst RNAi ovaries, or young ovaries were retained for further analysis ( $\mathrm{N}=9251$ genes). Tst regulated mRNAs were classified as genes whose transcript-per-million (TPM) expression levels were $>4$-fold increased in the tst RNAi samples; a subset of these that are additionally $>2$-fold increased in the tst mutant samples were considered to be strong Tst regulated mRNAs ( $N=207$ genes). To curate a set of non-target genes to serve as a background set, we identified genes that differed $<1.25$ fold between WT and tst $R N A i$ and selected the 3 non-targets with the most similar tst RNAi expression level to each Tst regulated $\mathrm{mRNA}$, to yield a set of 621 non-targets. For polysome profiling samples, normalized ribosome occupancy was calculated as $\log _{2}$ (polysome TPM / input TPM). To compare ribosome occupancy of Tst regulated mRNAs across different conditions relative to the global differences observed, we normalized Tst regulated mRNA ribosome occupancy by mean non-target occupancy, i.e. $\log _{2}(($ polysome_target TPM) / (input_target TPM) / average (polysome_non-target TPM / input_non-target TPM)). Codon optimality index (CAI) was calculated for each gene relative to the codon frequencies in the top 100 expressed genes in WT ovaries according to Sharp \& Li, $1987^{57}$.

\section{EdU}

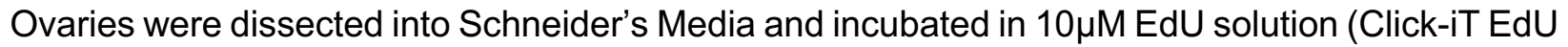
Flow Cytometry Assay Kit) rotating for one hour ${ }^{78}$. Samples were fixed in $3.7 \%$ formaldehyde in PBS, rotating for 30 minutes. Fixative was then aspirated and samples were washed with $1 \mathrm{~mL}$ PBS for 10 minutes and permeabilized in $1 \mathrm{~mL}$ of permeabilization solution (1\% Triton X-100 in PBST) rotating for 20 minutes. Samples were then washed in $1 \mathrm{~mL}$ PBS rotating for 10 minutes. Click-iT reaction cocktail (PBS, $\mathrm{CuSO}_{4}$, Fluorescent dye azide and Reaction Buffer Additive) was made according to manufacturer's directions and added to each sample. Tubes were protected from light and rotated at room temperature for 30 minutes. Samples were then washed once with $1 \mathrm{~mL}$ of Click-iT reaction rinse buffer and once with $1 \mathrm{~mL}$ PBS. Ovary samples were then transitioned to the immunostaining protocol. 


\section{Fluorescent in situ Hybridization}

Ovaries were dissected in RNase free $1 X$ PBS and fixed in $1 \mathrm{~mL}$ of $5 \%$ formaldehyde rotating for 10 minutes. Samples were then washed three times for five minutes each in PT buffer (PBS, 0.1\% Triton X-100) and dehydrated in successive methanol washes for six minutes each $(30 \%, 50 \%$, $70 \%$ ). A final $100 \%$ methanol wash was carried out for 12 minutes. Samples were equilibrated to PT buffer by conducting successive methanol washes for six minutes each $(70 \%, 50 \%, 30 \%)$, followed by three PT washes of six minutes each. Ovaries were pre-hybridized for six minutes in $1 \mathrm{~mL}$ Wash buffer (10\% Deionized Formamide, 2X SSC in RNase Free $\mathrm{H}_{2} \mathrm{O}$ ). Alexa-488 fluorescent probe against $p g c$ was generated by Stellaris. Hybridization of probes was conducted at $32^{\circ} \mathrm{C}$, covered for $>16$ hours. Samples were then washed six times in Wash buffer for 2 minutes per wash. Samples were then washed twice in $1 \mathrm{~mL}$ Wash buffer for 30 minutes at $30^{\circ} \mathrm{C}$. Wash buffer was aspirated and incubated in Vectashield for 30 minutes before mounting. in situ experiments were repeated more than three times for control and experimental ovaries.

\section{RNAScope ${ }^{\mathrm{TM}}$ Assay}

We utilized a modified RNAscope procedure for Drosophila ovaries described previously ${ }^{79}$. Probes were designed and generated by Advanced Cell Diagnostics with specificity to target base pairs 29-1250 of blanks mRNA (accession number from NCBI: NM_139709.2), base pairs 11961693 of actin57B mRNA (NM_079076.4). Ovaries were dissected in RNase free 1X PBS and fixed in $1 \mathrm{~mL}$ of $5 \%$ formaldehyde rotating for 10 minutes. Samples were then washed three times for five minutes each in PT buffer (PBS, $0.1 \%$ Triton X-100) and dehydrated in successive methanol washes for six minutes each $(30 \%, 50 \%, 70 \%)$. A final $100 \%$ methanol wash was carried out for 12 minutes. Samples were equilibrated to PT buffer by conducting successive methanol washes for six minutes each $(70 \%, 50 \%, 30 \%)$, followed by three PT washes of six minutes each. Ovaries were pre-hybridized for six minutes in $1 \mathrm{~mL}$ of RNAScope Wash buffer (ACD, 310091). Hybridization of probes was conducted at $40^{\circ} \mathrm{C}$, covered for $>16$ hours. Samples were then washed three times in RNAscope wash buffer for 5 minutes per wash, fixed in $4 \%$ formaldehyde in $1 \mathrm{X}$ PBS at room temperature for 10 minutes and washed in buffer three times for 5 minutes each. Ovaries were incubated in a successive series of amplifier solutions (Amp). Amp 1 for at least 45 minutes at $40^{\circ} \mathrm{C}$, Amp 2 for 45 minutes at $40^{\circ} \mathrm{C}$, Amp 3 for 45 minutes at $40^{\circ} \mathrm{C}$, Amp 4 for 45 minutes at $40^{\circ} \mathrm{C}$. After each Amp step ovaries were washed in wash buffer 5 times for 3 minutes each at room temperature. Samples were then washed in $1 \mathrm{~mL}$ PBT for 5 minutes and mounted in Vectashield. RNAscope experiments were repeated more than three times for control and tst RNAi ovaries.

\section{Materials and reagents}

Fly food was made according to previously published procedures, and filled narrow vials (Fisherbrand Drosophila Vials; Fischer Scientific) to approximately $12 \mathrm{~mL}^{32}$.

\section{RNA Immuno-Precipitation (RIP)}

65 pairs of ovaries were dissected in $1 \mathrm{XPBS}^{32}$. After dissection, PBS was aspirated and $100 \mu \mathrm{l}$ of RIPA buffer (50 mM Tris pH 8.0, 1\% Triton X-100, 0.1\% sodium deoxycholate, $0.1 \%$ SDS, 140 $\mathrm{mM} \mathrm{NaCl}, 1 \mathrm{mM}$ EDTA, $1 \mathrm{mM}$ PMSF, 1 protease inhibitor pill per $50 \mathrm{ml}$ ) was added and the sample was homogenized. An additional $200 \mu \mathrm{l}$ of RIPA buffer was added to the lysate and mixed. The lysate was then centrifuged at $13,000 \mathrm{rpm}$ for 10 minutes at $4^{\circ} \mathrm{C}$. The supernatant was transferred to a new tube. $10 \%$ of the cleared homogenate was set aside as input, 4X SDS buffer was added the sample was heated at $95^{\circ} \mathrm{C}$ for 5 minutes and stored at $-20^{\circ} \mathrm{C}$ until Western analysis. An additional $10 \%$ of homogenate was used for RNA input, $100 \mu \mathrm{l}$ of TriZol was added, mixed and this sample was stored in $-80^{\circ} \mathrm{C}$. $40 \%$ of homogenate was used for the IgG control and the remaining $40 \%$ was used for RIP. The following antibodies were added to the lysate and incubated 
405

406

407

408

409

410

411

412

413

414

415

416

417

418

419

420

421

422

423

424

425

426

427

428

429

430

431

432

433

434

435

436

437

438

439

440

441

442

443

444

445

446

447

448

449

450

451

452

453

454

at $4^{\circ} \mathrm{C}$ for 3 hours; $1 \mu$ l of Rabbit anti-HA (abcam, 9110), $1 \mu$ of ChromePure Rabbit IgG (Jackson ImmunoResearch Labs). Protein A Dynabeads (Thermo Fisher Scientific) were separated into 15 $\mu \mathrm{l}$ aliquots for each sample and washed four times in $400 \mu \mathrm{l}$ of 1:10 diluted protease inhibitorcontaining Net2 buffer (50 mM Tris- $\mathrm{Cl}$ [pH 8.0], $150 \mathrm{mM} \mathrm{NaCl}, 10 \% \mathrm{NP}-40$ ) on a magnetic rack. The beads were then re-suspended in $100 \mu \mathrm{l}$ of Net2 buffer. After lysate incubation $25 \mu \mathrm{l}$ of washed beads was added to each sample and incubated overnight at $4^{\circ} \mathrm{C}$. Beads were washed six times with $500 \mu$ of $1: 10$ diluted Net2 buffer for 2 minutes each. Beads were then resuspended in $25 \mu \mathrm{l}$ of Net2 buffer. An aliquot of $10 \mu \mathrm{l}$ was used for Western Blot analysis. The remaining 15 $\mu \mathrm{l}$ was used for RNA extraction.

\section{Protein Purification}

The coding sequence of two adjacent RRMs of Hfp (Amino acids 110-326) was PCR amplified from cDNA to incorporate Nco1 and Kpn1 sites and ligated into the pETM-82 expression plasmid via Gibson assembly (NEB) ${ }^{32}$. The completed plasmid was transformed into Rosetta BL21 cells (Millipore Sigma, 70954-3). A starter culture of $5 \mathrm{ml}$ of Rosetta BL21 cells containing the completed plasmid were grown overnight at $37^{\circ} \mathrm{C}$ in LB with Kanamycin. This culture was then added to $1000 \mathrm{~mL}$ of LB-Kan media. Cells were shaken at $220 \mathrm{rpm}$ at $37^{\circ} \mathrm{C}$ for 3 hours until OD600 0.6. To induce protein expression, $0.5 \mathrm{mM}$ IPTG was added to the culture and then shaken at $220 \mathrm{rpm}$ at $37^{\circ} \mathrm{C}$ for 3 hours. The cells were then centrifuged at $4000 \mathrm{xg}$ for 20 minutes at $4^{\circ} \mathrm{C}$ in $50 \mathrm{~mL}$ aliquots. The pellet was re-suspended in $3 \mathrm{~mL}$ of re-suspension buffer $(20 \mathrm{mM}$

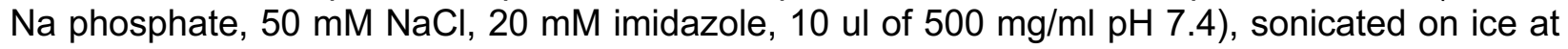
$20 \%$ intensity for 20 seconds for 3 pulses using 1/8-inch probe. The suspension was then centrifuged at $10,000 x g$ for 10 minutes at $4^{\circ} \mathrm{C}$. The column (His GraviTrap, GE Cat\#11-0033-99) was equilibrated with $10 \mathrm{~mL}$ binding buffer $(20 \mathrm{mM}$ Na phosphate, $50 \mathrm{mM} \mathrm{NaCl}, 20 \mathrm{mM}$ imidazole,

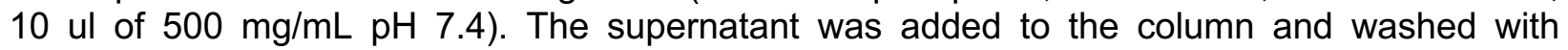
increments of $1 \mathrm{~mL}, 4 \mathrm{~mL}$ and $5 \mathrm{~mL}$ of binding buffer. The protein was then eluted using the following washes; twice with $1 \mathrm{~mL}$ of elution buffer \#1, twice with $1 \mathrm{~mL}$ of elution buffer \#2 and three times with $1 \mathrm{~mL}$ of elution buffer \#3. Elution Buffer \#1: $20 \mathrm{mM} \mathrm{NaPO}$, $50 \mathrm{mM} \mathrm{NaCl}, 150$ $\mathrm{mM}$ imidazole, pH 7.4 Elution Buffer \#2: 20 mM NaPO4, 50 mM NaCl, 300 mM imidazole, pH 7.4 Elution Buffer \#3: $20 \mathrm{mM}$ NaPO4, $50 \mathrm{mM} \mathrm{NaCl}, 500 \mathrm{mM}$ imidazole, $\mathrm{pH} 7.4$ The first elution contained purified Hfp RRM protein. The eluted protein was de-salted using the PD-10 column (GE \#17-0851-01). The column was equilibrated with $25 \mathrm{~mL}$ of binding buffer (10 mM HEPES, 150 $\mathrm{mM} \mathrm{KCl}, \mathrm{pH} 7.5)$ and centrifuged at $3500 \mathrm{rpm}$ for 2 minutes. The eluted protein was slowly added to the column and centrifuged at $3500 \mathrm{rpm}$ for 2 minutes. Desalted protein concentration was determined by Bradford assay. The eluted protein was then stored in $20 \%$ glycerol at $-80^{\circ} \mathrm{C}$ until use.

\section{Electrophoretic Mobility Shift Assay (EMSA)}

Positive control oligo: 5'-UUUUUCUCUU-3', negative control scramble: 5'-UACGUACGUA-3', act5C sequence: 5'-UCUUCCCCAUC-3', act57B sequence: 5'-UCUUCCCCUC-3' RNA oligonucleotides were end-labeled using T4 Kinase (NEB) with ATP $[\mathrm{Y}-32 \mathrm{P}]^{32}$. Excess ATP was removed through G-25 Sephadix Columns (Roche, 11273990001). RNA-binding reactions were performed in 1X Binding Buffer $(50 \mathrm{mM}$ Tris $\mathrm{pH} 7.5,150 \mathrm{mM} \mathrm{NaCl}, 2 \mathrm{mM}$ DTT, $0.1 \mathrm{mg} / \mu \mathrm{l} B S A$, $0.001 \%$ Tween-20, $0.5 \mu$ l of dldC, $1 \mu \mathrm{l}$ RNaseOUT and $0.5 \mu l$ of yeast t-RNA) ${ }^{80}$. $3.0 \mathrm{nM}$ of RNA oligo and 3.6 $\mu \mathrm{M}$ purified Hfp RRM protein was incubated for 20 minutes at RT and then ran on an $3.5 \%$ native polyacrylamide TBE gel at $150 \mathrm{~V}$ for 4 hours at $4^{\circ} \mathrm{C}$. The gel was then dried onto Whatmann filter paper for one hour and exposed to a phosphor screen overnight. A Typhoon Trio imager was used to image the phosphor screen.

\section{Subcellular Fractionation}


455

456

457

458

459

460

461

462

463

464

465

466

467

468

469

470

471

472

473

474

475

476

477

478

479

480

481

482

483

484

485

486

487

488

489

490

491

492

493

494

495

496

497

498

499

500

501

502

503

50 adult Wild Type ovaries were dissected in $1 \mathrm{X}$ PBS and homogenized with 10-20 strokes of a plastic homogenizer in $100 \mu \mathrm{L}$ hypotonic lysis buffer $(10 \mathrm{mM} \mathrm{HEPES} \mathrm{pH} 7.9,1.5 \mathrm{mM} \mathrm{MgCl} 2,10$ $\mathrm{mM} \mathrm{KCl}, 0.5 \mathrm{mM}$ DTT). Homogenate was incubated on ice for 15 minutes. $50 \mu \mathrm{L}$ of homogenate was aliquoted in a new tube, 4X SDS buffer was added, sample was boiled at $95^{\circ} \mathrm{C}$ for 5 minutes and stored in $-20^{\circ} \mathrm{C}$ until use as total homogenate. The remaining homogenate was centrifuged for 10 minutes at $1000 \mathrm{~g} .50 \mu \mathrm{L}$ of supernatant was collected 4 X SDS buffer was added, sample was boiled at $95^{\circ} \mathrm{C}$ for 5 minutes and stored in $-20^{\circ} \mathrm{C}$ until use as cytoplasmic fraction. The pellet was resuspended in high salt extraction buffer (20mM HEPES pH 7.9, 25\% glycerol, $420 \mathrm{mM}$ $\mathrm{NaCl}, 1.5 \mathrm{mM} \mathrm{MgCl}_{2}, 0.2 \mathrm{mM}$ EDTA, $0.5 \mathrm{mM}$ DTT) and centrifuged for 5 minutes at 20,000g. Supernatant was collected $4 \mathrm{X}$ SDS buffer was added, sample was boiled at $95^{\circ} \mathrm{C}$ for 5 minutes and stored in $-20^{\circ} \mathrm{C}$ until use as total nuclear fraction.

\section{Western Blot}

Twenty wild-type size ovaries or 40 mutant size ovaries were dissected in $1 \mathrm{X} \mathrm{PBS}^{32}$. After dissection, PBS was aspirated and $30 \mu \mathrm{l}$ of NP-40 buffer with protease inhibitors added to the tissue and homogenized. The lysate was centrifuged at $13,000 \mathrm{rpm}$ for 15 minutes at $4^{\circ} \mathrm{C}$. Aqueous layer was transferred into a new tube while avoiding the top lipid layer. $1 \mu$ l of the protein extract was used to carry out a Bradford (Bio-Rad, 500-0205) assay. $25 \mu \mathrm{g}$ of protein was denatured with $4 \mathrm{X}$ Laemmli Sample Buffer (Bio-Rad, 161-0747) and $\beta$-marcepthanol at $95^{\circ} \mathrm{C}$ for 5 minutes. The samples were loaded in a Mini-PROTEAN TGX 4-20\% gradient SDS-PAGE gels (Bio-Rad, 456-1094) and run at 110V for 1 hour. The proteins were then transferred to a $0.20 \mu \mathrm{m}$ nitrocellulose membrane at $100 \mathrm{~V}$ for 1 hour at $4^{\circ} \mathrm{C}$. After transfer, the membrane was blocked in $5 \%$ milk in PBST for 2 hours at RT. The following antibodies were used: Mouse anti-Hfp (1:1000, Schüpbach Lab), Rabbit anti-Orb (1:1000, Lehmann Lab), Rabbit anti-His (1:000, Rockland Inc., 600-401-382), Mouse anti-Fibrillarin (1:25, DSHB). Primary antibody was prepared in $5 \%$ milk in PBST was added to the membrane and incubated at $4^{\circ} \mathrm{C}$ overnight. The membrane was then washed three times in 0.5\% milk PBST. Anti-Rabbit HRP (1:10,000, Abcam, ab97046) or AntiMouse HRP $(1: 10,000$, Abcam, ab6721) was prepared in 5\% milk in PBST, and was added to the membrane and incubated at room temperature for 2 hours. The membrane was then washed 3 times in PBST. Bio-rad chemiluminescence ECL kit (1705061) was used to image the membrane.

\section{Egg Laying Assay}

Newly eclosed flies were collected and fattened overnight on yeast. Assays were conducted in cages on apple juice plates containing 6 control or experimental females crossed to 4 Wild Type control males. Cages were maintained at $25^{\circ} \mathrm{C}$ and plates changed daily for counting. Analyses were performed on three consecutive days. Total number of eggs laid was counted and averaged. Both control and experimental experiments were conducted in triplicate.

\section{Polysome profiling and Polysome-Seq}

30 Wild Type or 150 experimental ovary pairs were dissected in 1X PBS and immediately flash frozen on liquid nitrogen 32,81 . Samples were homogenized in lysis buffer and $20 \%$ of lysate was used as input for mRNA isolation and library preparation (as described above). Samples were loaded onto 10-45\% CHX supplemented sucrose gradients in 9/16 x 3.5 PA tubes (Beckman Coulter, \#331372) and spun at 35,000 x g in SW41 for 3 hours at $4^{\circ} \mathrm{C}$. Gradients were fractionated with a Density Gradient Fractionation System (\#621140007). RNA was extracted using acid phenol-chloroform and precipitated overnight. Pelleted RNA was resuspended in $20 \mu \mathrm{L}$ water, treated with TURBO DNase and libraries were prepared as described above.

\section{MEME Analyses}


The 5'UTR, CDS, 3'UTR and full transcript sequences of all 207 Tst-regulated target genes were individually analyzed by the MEME algorithm ${ }^{82}$. Classic mode analysis was utilized to conduct de novo motif search with default parameters as well as Any Number of Repetitions (anr) mode. Discriminative mode analysis was conducted against 621 non-target gene sequences as background with default parameters. Motif logos, number of sites, and p-values all reported as produced by output of the program.

\section{Acknowledgements}

We would like to thank all members of the Rangan Lab as well as Dr. Sano $\mathrm{H}$, Juliano C, Belfort $\mathrm{M}$, and Farrell $\mathrm{J}$ for discussion and comments on the manuscript. We would also like to thank the Schüpbach Lab for the Hfp antibody and mutant flies, the Sontheimer Lab for the Blanks antibody, and the Newbury Lab for flies and reagents. P.R. is funded by the NIH/NIGMS (R01GM11177906). P.B. is funded by NIH (grant 1F31GM126784-01) and by the RNA Institute. M.T.L. was supported by start-up funds from the Univ. of Pittsburgh.

\section{References:}

1. Cinalli, R. M., Rangan, P. \& Lehmann, R. Germ cells are forever. Cell 132, 559-562 (2008).

2. Seydoux, G. \& Braun, R. E. Pathway to Totipotency: Lessons from Germ Cells. Cell 127, 891-904 (2006).

3. Reik, W. \& Surani, M. A. Germline and Pluripotent Stem Cells. Cold Spring Harbor Perspectives in Biology 7, (2015).

4. Walser, C. B. \& Lipshitz, H. D. Transcript clearance during the maternal-to-zygotic transition. Current Opinion in Genetics \& Development 21, 431-443 (2011).

5. Laver, J. D., Marsolais, A. J., Smibert, C. A. \& Lipshitz, H. D. Regulation and Function of Maternal Gene Products During the Maternal-to-Zygotic Transition in Drosophila. Current topics in developmental biology 113, 43-84 (2015).

6. Lee, M. T., Bonneau, A. R. \& Giraldez, A. J. Zygotic Genome Activation During the Maternal-to-Zygotic Transition. Annu. Rev. Cell Dev. Biol. 30, 581-613 (2014).

7. Lehmann, R. Germline stem cells: Origin and destiny. Cell Stem Cell 10, 729-739 (2012).

8. Spradling, A. C. et al. The Drosophila germarium: stem cells, germ line cysts, and oocytes. Cold Spring Harbor Symposia on Quantitative Biology 62, 25-34 (1997).

9. Xie, T. \& Spradling, A. C. decapentaplegic is essential for the maintenance and division of germline stem cells in the Drosophila ovary. Cell 94, 251-260 (1998).

10. Spradling, A., Drummond-Barbosa, D. \& Kai, T. Stem cells find their niche. Nature 414, 98-104 (2001).

11. McKearin, D. M. \& Spradling, A. C. bag-of-marbles: a Drosophila gene required to initiate both male and female gametogenesis. Genes \& Development 4, 2242-2251 (1990).

12. Eichhorn, S. W. et al. mRNA poly(A)-tail changes specified by deadenylation broadly reshape translation in Drosophila oocytes and early embryos. eLife 5, 1-24 (2016).

13. Navarro, C., Puthalakath, H., Adams, J. M., Strasser, A. \& Lehmann, R. Egalitarian binds dynein light chain to establish oocyte polarity and maintain oocyte fate. Nat Cell Biol 6, 427-435 (2004).

14. Huynh, J.-R. \& St Johnston, D. The origin of asymmetry: early polarisation of the Drosophila germline cyst and oocyte. Current Biology 14, R438-49 (2004).

15. Lantz, V., Chang, J. S., Horabin, J. I., Bopp, D. \& Schedl, P. The Drosophila orb RNAbinding protein is required for the formation of the egg chamber and establishment of polarity. Genes \& Development 8, 598-613 (1994).

16. Kugler, J.-M. \& Lasko, P. Localization, anchoring and translational control of oskar, gurken, bicoid and nanos mRNA during drosophila oogenesis. Fly 3, 15-28 (2009). 
17. Spradling, A. C. \& Mahowald, A. P. Amplification of genes for chorion proteins during oogenesis in \&lt;em\&gt;Drosophila melanogaster\&lt;/em\&gt. Proc. Natl Acad. Sci. USA 77, 1096 (1980).

18. Navarro-Costa, P. et al. Early programming of the oocyte epigenome temporally controls late prophase i transcription and chromatin remodelling. Nat Comms 7, (2016).

19. Seago, J. E., Chernukhin, I. V. \& Newbury, S. F. The Drosophila gene twister, an orthologue of the yeast helicase SKI2, is differentially expressed during development. Mechanisms of Development 106, 137-141 (2001).

20. Anderson, J. S. \& Parker, R. P. The 39 to 59 degradation of yeast mRNAs is a general mechanism for mRNA turnover that requires the SKI2 DEVH box protein and 39 to 59 exonucleases of the exosome complex. Embo J 17, 1497-1506 (1998).

21. Halbach, F., Rode, M. \& Conti, E. The crystal structure of S. cerevisiae Ski2, a DExH helicase associated with the cytoplasmic functions of the exosome. RNA 18, 124-134 (2012).

22. Schmidt, C. et al. The cryo-EM structure of a ribosome-Ski2-Ski3-Ski8 helicase complex. Science 354, 1431-1433 (2016).

23. Tanentzapf, G., Devenport, D., Godt, D. \& Brown, N. H. Integrin-dependent anchoring of a stem-cell niche. Nat Cell Biol 9, 1413-1418 (2007).

24. Doren, M. V., Williamson, A. L. \& Lehmann, R. Regulation of zygotic gene expression in Drosophila primordial germ cells. Current Biology 8, 243-246 (1998).

25. Halbach, F., Reichelt, P., Rode, M. \& Conti, E. The Yeast Ski Complex: Crystal Structure and RNA Channeling to the Exosome Complex. Cell 154, 814-826 (2013).

26. Théry, C., Zitvogel, L. \& Amigorena, S. Exosomes: composition, biogenesis and function. Nat Rev Immunol 2, 569-579 (2002).

27. Becker, T. et al. Structure of the no-go mRNA decay complex Dom34-Hbs1 bound to a stalled 80 S ribosome. Nat Struct Mol Biol 18, 715-720 (2011).

28. Yang, F. et al. The RNA surveillance complex Pelo-Hbs1 is required for transposon silencing in the Drosophila germline. EMBO reports 16, 965-974 (2015).

29. Li, Z., Yang, F., Xuan, Y., Xi, R. \& Zhao, R. Pelota-interacting G protein Hbs1 is required for spermatogenesis in Drosophila. Sci Rep 1-14 (2019). doi:10.1038/s41598-01939530-6

30. Théry, C., Zitvogel, L. \& Amigorena, S. Exosomes: composition, biogenesis and function. arXiv 2, 569-579 (2002).

31. Halbach, F. Structural and Functional Characterization of the Yeast Ski2-Ski3-Ski8 Complex. 1-126 (2013).

32. Flora, P. et al. Sequential Regulation of Maternal mRNAs through a Conserved cis-Acting Element in Their 3' UTRs. CellReports 25, 3828-3843.e9 (2018).

33. Ohlstein, B. \& McKearin, D. Ectopic expression of the Drosophila Bam protein eliminates oogenic germline stem cells. Development 124, 3651 (1997).

34. Jia Ng, S. S., Zheng, R. T., Osman, I. \& Pek, J. W. Generation of Drosophila sisRNAs by Independent Transcription from Cognate Introns. ISCIENCE 4, 68-75 (2018).

35. Wineland, D. M., Kelpsch, D. J. \& Tootle, T. L. Multiple Pools of Nuclear Actin. Anat. Rec. 301, 2014-2036 (2018).

36. Duan, T., Green, N., Tootle, T. L. \& Geyer, P. K. Nuclear architecture as an intrinsic regulator of Drosophila female germline stem cell maintenance. Development and regulation 37, 30-38 (2020).

37. Kelpsch, D. J., Groen, C. M., Fagan, T. N., Sudhir, S. \& Tootle, T. L. Fascin regulates nuclear actin during Drosophila oogenesis. Molecular Biology of the Cell 27, 2965-2979 (2016).

38. Fingerhut, J. M., Moran, J. V. \& Yamashita, Y. M. Satellite DNA-containing gigantic introns in a unique gene expression program during Drosophila spermatogenesis. PLOS 
606

607

608

609

610

611

612

613

614

615

616

617

618

619

620

621

622

623

624

625

626

627

628

629

630

631

632

633

634

635

636

637

638

639

640

641

642

643

644

645

646

647

648

649

650

651

652

653

654

655

656

Genet 15, e1008028 (2019).

39. Estes, J. E., Selden, L. A. \& Gershman, L. C. Mechanism of action of phalloidin on the polymerization of muscle actin. Biochemistry 20, 708-712 (1981).

40. Schneider, C. \& Tollervey, D. Threading the barrel of the RNA exosome. 1-9 (2013). doi:10.1016/j.tibs.2013.06.013

41. Avery, P. et al. Drosophila Upf1 and Upf2 loss of function inhibits cell growth and causes animal death in a Upf3-independent manner. RNA 17, 624-638 (2011).

42. Mitchell, P. \& Tollervey, D. An NMD pathway in yeast involving accelerated deadenylation and exosome-mediated 3' $\rightarrow 5^{\prime}$ degradation. Molecular Cell 11, 1405-1413 (2003).

43. Lykke-Andersen, S. \& Jensen, T. H. Nonsense-mediated mRNA decay: an intricate machinery that shapes transcriptomes. Nature Publishing Group 16, 665-677 (2015).

44. Peltz, S. W., Brown, A. H. \& Jacobson, A. mRNA destabilization triggered by premature translational termination depends on at least three cis-acting sequence elements and one trans-acting factor. Genes \& Development 7, 1737-1754 (1993).

45. Dunn, J. G., Foo, C. K., Belletier, N. G., Gavis, E. R. \& Weissman, J. S. Ribosome profiling reveals pervasive and regulated stop codon readthrough in Drosophila melanogaster. eLife 2013, 1-32 (2013).

46. Hashimoto, Y., Takahashi, M., Sakota, E. \& Nakamura, Y. Nonstop-mRNA decay machinery is involved in the clearance of mRNA 5'-fragments produced by RNAi and NMD in Drosophila melanogaster cells. Biochemical and Biophysical Research Communications 484, 1-7 (2017).

47. Schweingruber, C., Rufener, S. C., Zünd, D., Yamashita, A. \& Mühlemann, O. Nonsensemediated mRNA decay - Mechanisms of substrate mRNA recognition and degradation in mammalian cells. BBA - Gene Regulatory Mechanisms 1829, 612-623 (2013).

48. Kobayashi, K. et al. Structural basis for mRNA surveillance by archaeal Pelota and GTPbound EF1alpha complex. Proc. Natl. Acad. Sci. U.S.A. 107, 17575-17579 (2010).

49. Jamar, N. H., Kritsiligkou, P. \& Grant, C. M. The non-stop decay mRNA surveillance pathway is required for oxidative stress tolerance. Nucleic Acids Research 45, 68816893 (2017).

50. Xi, R., Doan, C., Liu, D. \& Xie, T. Pelota controls self-renewal of germline stem cells by repressing a Bam-independent differentiation pathway. Development 132, 5365-5374 (2005).

51. Edri, S. \& Tuller, T. Quantifying the Effect of Ribosomal Density on mRNA Stability. PLoS ONE 9, e102308 (2014).

52. Guydosh, N. R. \& Green, R. Dom34 Rescues Ribosomes in 3' Untranslated Regions. Cell 156, 950-962 (2014).

53. Guydosh, N. R. \& Green, R. Translation of poly(A) tails leads to precise mRNA cleavage. RNA 23, 749-761 (2017).

54. Zinoviev, A., Ayupov, R. K., Abaeva, I. S., Hellen, C. U. T. \& Pestova, T. V. Extraction of mRNA from Stalled Ribosomes by the Ski Complex. Molecular Cell 77, 1340-1349.e6 (2020).

55. Simms, C. L., Yan, L. L. \& Zaher, H. S. Ribosome Collision Is Critical for Quality Control during No-Go Decay. Molecular Cell 68, 361-373.e5 (2017).

56. Hanson, G. \& Coller, J. Codon optimality, bias and usage in translation and mRNA decay. Nat Rev Mol Cell Biol (2017). doi:10.1038/nrm.2017.91

57. Sharp, P. M. \& Li, W. H. The codon Adaptation Index--a measure of directional synonymous codon usage bias, and its potential applications. Nucleic Acids Research 15, 1281-1295 (1987).

58. Presnyak, V. et al. Codon optimality is a major determinant of mRNA stability. Cell 160, 1111-1124 (2015).

59. Serano, T. L., Cheung, H.-K., Frank, L. H. \& Cohen, R. S. P element transformation 
vectors for studying Drosophila melanogaster oogenesis and early embryogenesis. Gene 138, 181-186 (1994).

60. Gavis, E. R. \& Lehmann, R. Localization of nanos RNA controls embryonic polarity. Cell 71, 301-313 (1992).

61. Gavis, E. R. \& Lehmann, R. Translational regulation of nanos by RNA localization. Nature 369, 315-318 (1994).

62. McDermott, S. M. \& Davis, I. Drosophila Hephaestus/polypyrimidine tract binding protein is required for dorso-ventral patterning and regulation of signalling between the germline and soma. PLoS ONE 8, e69978-e69978 (2013).

63. Van Buskirk, C. \& Trudi, S. Half pint Regulates Alternative Splice Site Selection in Drosophila. 1-11 (2002).

64. Chen, D. \& McKearin, D. Dpp signaling silences bam transcription directly to establish asymmetric divisions of germline stem cells. Current Biology 13, 1786-1791 (2003).

65. Chen, D. \& Mckearin, D. M. A discrete transcriptional silencer in the bam gene determines asymmetric division of the Drosophila germline stem cell. Development (Cambridge, England) 130, 1159-1170 (2003).

66. Lilly, M. A. \& Sptadling, A. C. The Drosophila endocycle is controlled by cyclin E and lacks a checkpoint ensuring S-phase completion. Genes \& Development 10, 2514-2526 (1996).

67. Calvi, B. R., Lilly, M. A. \& Spradling, A. C. Cell cycle control of chorion gene amplification. Genes \& Development 12, 734-744 (1998).

68. Mach, J. M. \& Lehmann, R. An Egalitarian-BicaudalD complex is essential for oocyte specification and axis determination in Drosophila. Genes \& Development 11, 423-435 (1997).

69. Schultz, R. M. The molecular foundations of the maternal to zygotic transition in the preimplantation embryo. Hum Reprod Update 8, 323-331 (2002).

70. Tadros, W. et al. Regulation of maternal transcript destabilization during egg activation in Drosophila. Genetics 164, 989-1001 (2003).

71. Cabrera Quio, L. E., Schleiffer, A., Mechtler, K. \& Pauli, A. Zebrafish Ski7 tunes RNA levels during the oocyte-to-embryo transition. bioRxiv 2020.03.19.998716 (2020).

72. Xie, T. \& Spradling, A. C. A Niche Maintaining Germ Line Stem Cells in the Drosophila Ovary. Science Reports 290, 328-330 (2000).

73. Gerbasi, V. R. et al. Blanks, a nuclear siRNA/dsRNA-binding complex component, is required for Drosophila spermiogenesis. Proceedings of the National Academy of Sciences 108, 3204-3209 (2011).

74. Upadhyay, M. et al. Transposon Dysregulation Modulates dWnt4 Signaling to Control Germline Stem Cell Differentiation in Drosophila. PLoS Genet 12, (2016).

75. McCarthy, A., Deiulio, A., Martin, E. T., Upadhyay, M. \& Rangan, P. Tip60 complex promotes expression of a differentiation factor to regulate germline differentiation in female Drosophila. Molecular Biology of the Cell 29, 2933-2945 (2018).

76. Kim, D., Langmead, B. \& Salzberg, S. L. HISAT: a fast spliced aligner with low memory requirements. Nat Meth 12, 357-360 (2015).

77. Liao, Y., Smyth, G. K. \& Shi, W. featureCounts: an efficient general purpose program for assigning sequence reads to genomic features. Bioinformatics 30, 923-930 (2014).

78. Flora, P. et al. Transient transcriptional silencing alters the cell cycle to promote germline stem cell differentiation in Drosophila. Developmental Biology 434, 84-95 (2018).

79. Wang, X. \& Page-McCaw, A. Wnt6 maintains anterior escort cells as an integral component of the germline stem cell niche. Development (Cambridge, England) 145, (2018).

80. Weidmann, C. A. et al. Drosophila Nanos acts as a molecular clamp that modulates the RNA-binding and repression activities of Pumilio. eLife 5, e17096 (2016). 
bioRxiv preprint doi: https://doi.org/10.1101/2020.06.30.179986; this version posted July 2, 2020. The copyright holder for this preprint (which

was not certified by peer review) is the author/funder, who has granted bioRxiv a license to display the preprint in perpetuity. It is made available under aCC-BY-NC-ND 4.0 International license.

708

709

710

711

712

713

714

715

716

717

718

719

720

721

722

723

724

725

726

727

728

729

730

731

732

733

734

735

736

737

81. Fuchs, G., Diges, C., Kohlstaedt, L. A., Wehner, K. A. \& Sarnow, P. Proteomic analysis of ribosomes: translational control of mRNA populations by glycogen synthase GYS1. Journal of Molecular Biology 410, 118-130 (2011).

82. Bailey, T. L. \& Elkan, C. Fitting a mixture model by expectation maximization to discover motifs in biopolymers. Proceedings of the Second International Conference on Intelligent Systems for Molecular Biology 2, 28-36 (1994). 

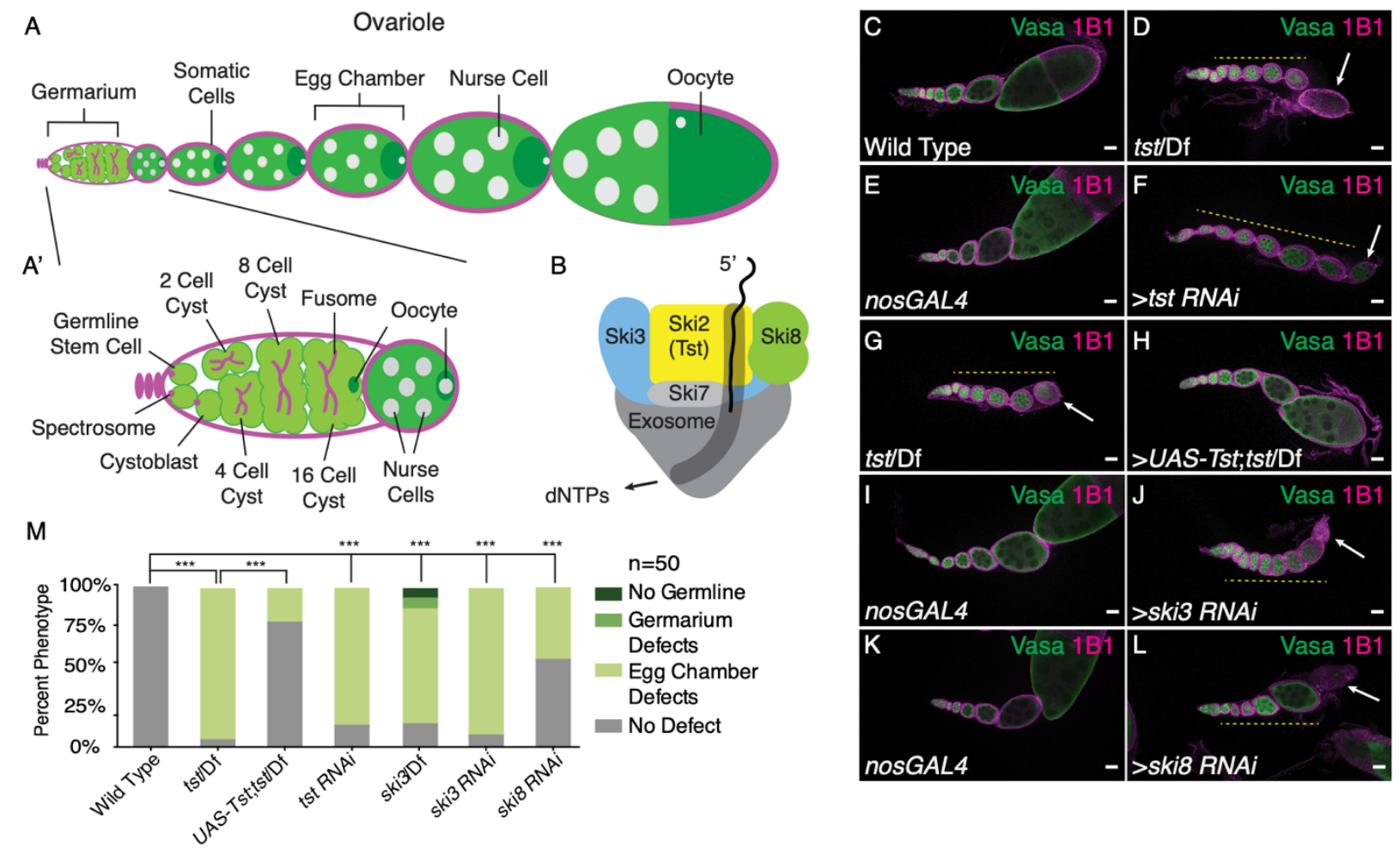

Figure 1. Components of the Ski Complex are required in the germ line for oogenesis. (A) Schematic of a Drosophila ovariole and (A') germarium. (B) Representation of the Ski Complex composed of Ski2 (Tst, yellow), Ski3 (blue), two Ski8 proteins (green), and Ski7 (light gray) threading mRNA (black) into the exosome (dark gray) where mRNA degradation occurs. (C) Confocal image of an adult WT control ovariole stained with Vasa (green) and 1B1 (magenta) showing normal egg chamber development. (D) Confocal image of a tst genomic mutant ovariole stained with 1B1, Vasa and indicating egg chambers that do not grow in size (yellow dashed line) and dying egg chamber (arrow). (E) Confocal image of a nosGAL4 driver control ovariole stained with 1B1 and Vasa. (F) tst germline RNAi knockdown ovariole stained with 1B1, Vasa, and indicating egg chambers that do not grow in size (yellow dashed line) and dying egg chamber (arrow). (G) Confocal image of a tst genomic mutant ovariole stained with 1B1 and Vasa. (H) Confocal image of a tst genomic mutant ovariole expressing recombinant Tst protein in the germline stained with $1 \mathrm{~B} 1$ and Vasa. (I) Confocal image of a nosGAL4 driver control ovariole stained with 1B1 and Vasa. (J) ski3 germline RNAi knockdown ovariole of stained with 1B1, Vasa, and indicating egg chambers that do not grow in size (yellow dashed line) and dying egg chamber (arrow). (K) Confocal image of a nosGAL4 driver control ovariole stained with 1B1 and Vasa. (L) ski8 germline RNAi knockdown ovariole stained with 1B1, Vasa, and indicating egg chambers that do not grow in size (yellow dashed line) and dying egg chamber (arrow). (M) Quantification of oogenesis defect phenotypes observed in Ski complex genomic mutants, germline RNAi knockdowns and UAS-Tst rescue (Control vs tst/Df $n=50, p<0.001$, tst/Df vs UAS-Tst; tst/Df $n=50$, $\mathrm{p}<0.001$, Control vs tst RNAi $\mathrm{n}=50, \mathrm{p}<0.001$, Control vs ski3/Df $\mathrm{n}=50, \mathrm{p}<0.001$, Control vs ski3

761

762

763 $10 \mu \mathrm{m}$. 
A

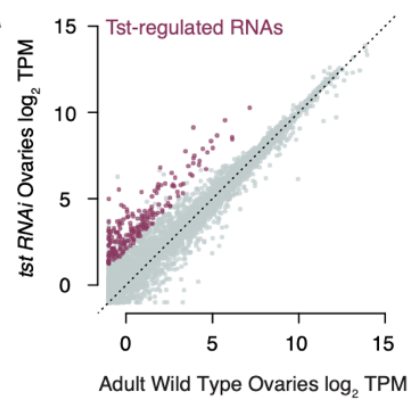

B

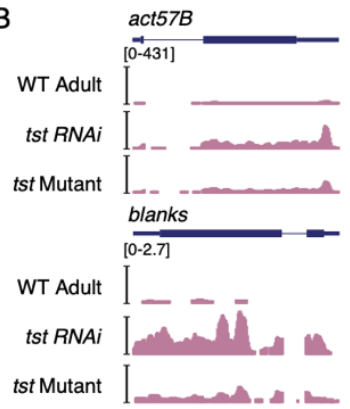

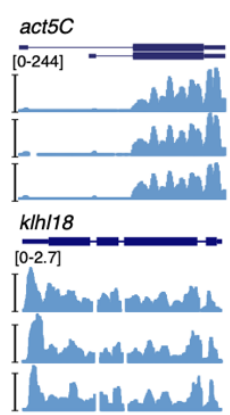

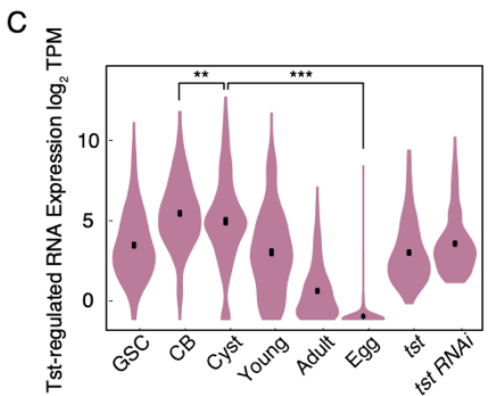

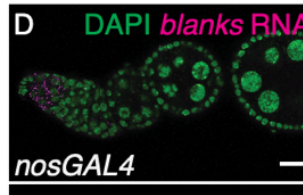
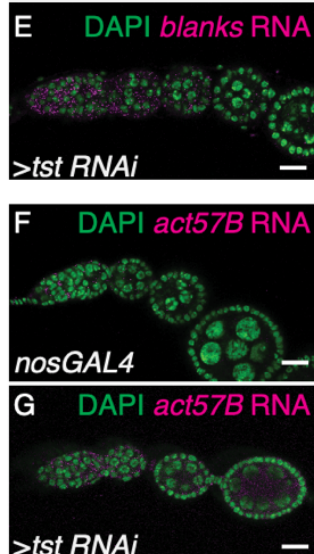
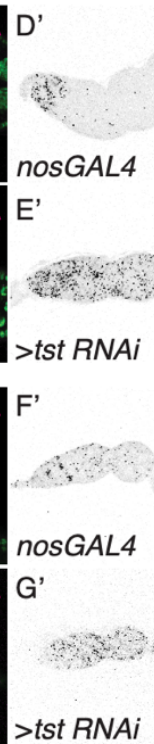

blanks RNA H
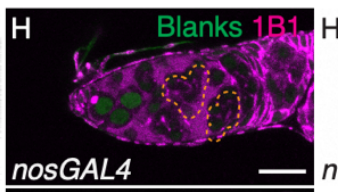

nosGAL4

blanks RNA
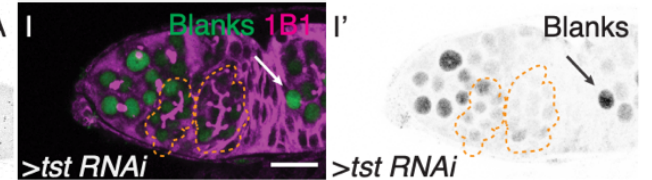

C4 $M$

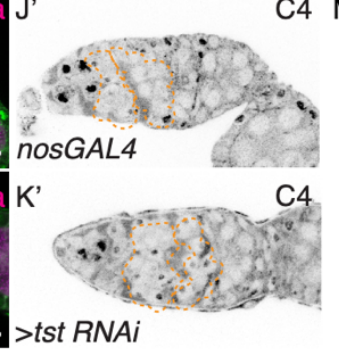

764

765

766

767

768

769

770

771

772

773

774

775

776

777

778

779

780

781

782

783

784

785

786

Figure 2. Tst promotes degradation of a subset of transcripts prior to oocyte specification. (A) Biplot of RNA-Seq data from adult WT and tst germline RNAi knockdown ovaries in $\log _{2}$ Transcripts Per Million (TPM) highlighting upregulated Tst-regulated RNAs (magenta). (B) Genome browser tracks of Tst-regulated genes act57B and blanks (magenta) and non-target genes act5C and klh/18 (blue). (C) Violin plot of Tst-regulated RNAs from germline stem cells (GSC), cystoblasts (CB), differentiating cysts (Cyst), young WT ovaries (Young), adult WT ovaries (Adult), unfertilized eggs (Egg), tst mutant (tst) and tst germline RNAi (tst RNAi) ovaries showing the decrease in expression of Tst-regulated RNAs after differentiation and cyst stages ( $n=207$, CB vs cyst $p<0.002$, cyst vs egg $p<0.0001$, Paired t-Test). (D-D') Confocal images of in situ hybridizations probing against blanks RNA (magenta, grayscale) and DAPI (green) in nosGAL4 showing blanks RNA expression restricted to the undifferentiated cells and in (E-E') tst RNAi ovarioles where blanks RNA expression is expanded to egg chambers. (F-F') Confocal images of in situ hybridizations probing against act57B mRNA (magenta, grayscale) and DAPI (green) in nosGAL4 showing low act57B RNA expression and (G-G') tst RNAi ovarioles exhibiting expanded act57B RNA expression in the germarium and egg chambers. (H-H') Confocal images of nosGAL4 and (I-I') tst RNAi germaria stained for 1B1 (magenta) and Blanks protein (green and grayscale) showing expanded Blanks expression in tst RNAi cysts (orange dashed lines) and egg chambers (arrow). (J-J') Confocal images of nosGAL4 and (K-K') tst RNAi germaria stained for Vasa (magenta) and $\mathrm{C} 4$ antibody (nuclear Actin) (green and grayscale) showing expanded nuclear Actin expression in tst RNAi cysts (orange dashed line). (L) Arbitrary Units (A.U.) 
quantification of Blanks protein expression normalized to control cysts in Control (gray) and tst RNAi (magenta) cysts and egg chambers (WT cyst $\mathrm{n}=60$, tst RNAi cyst $\mathrm{n}=70, \mathrm{p}<0.0001$. WT egg chamber $\mathrm{n}=49$, tst $R N A$ i egg chamber $\mathrm{n}=102$, $\mathrm{p}<0.0001$, Student's t-Test). (M) A.U. quantification of nuclear Actin expression normalized to control cysts in Control (gray) and tst RNAi (magenta) cysts and egg chambers (WT cyst $\mathrm{n}=92$, tst RNAi cyst $\mathrm{n}=73, \mathrm{p}<0.0001$. WT egg chamber $\mathrm{n}=38$,
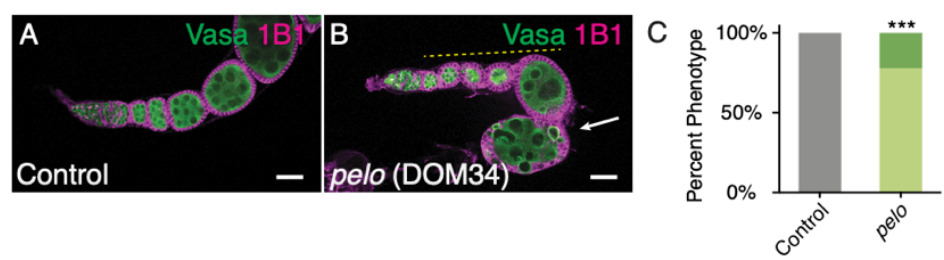

E

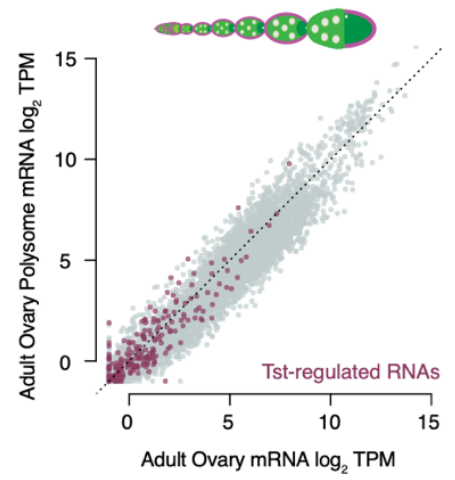

$\mathrm{H}$

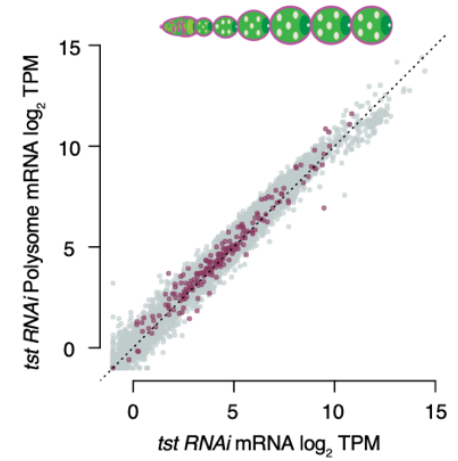

$\mathrm{F} \sum_{\mathrm{O}}$
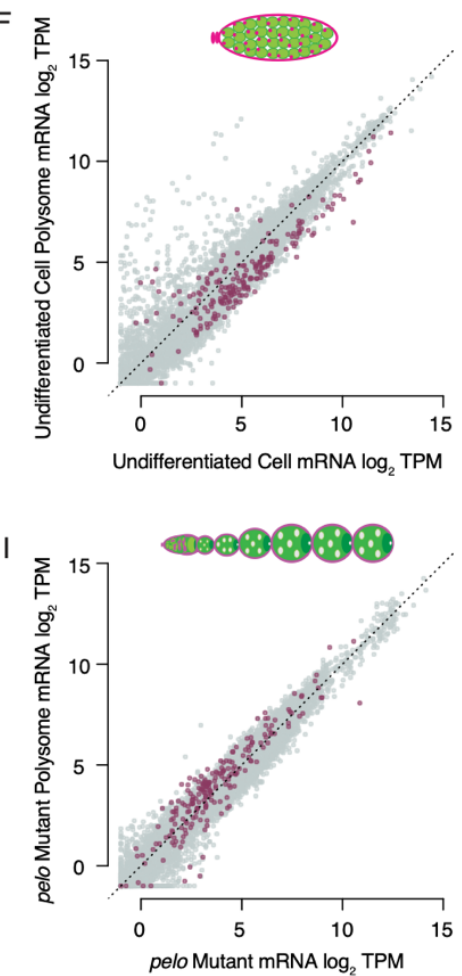

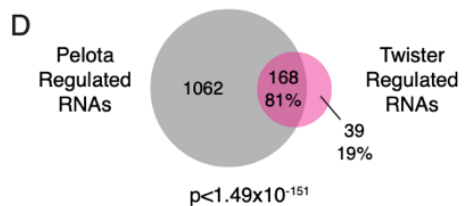

$\mathrm{G}$

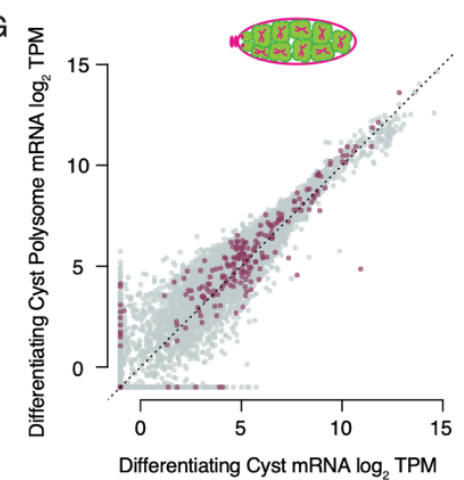

J

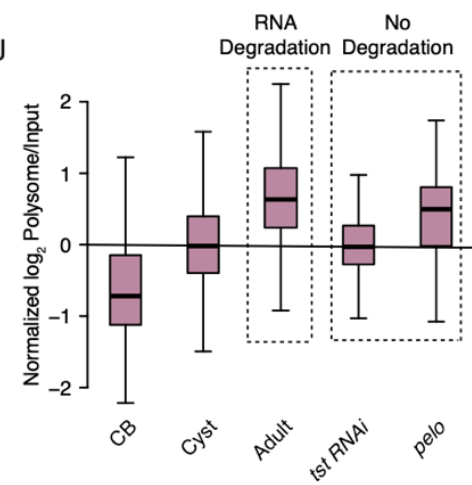

796

797

798

799

800

801

802

803

804

805

806

807

808

809

Figure 3. Tst-regulated RNAs are coregulated by Pelota and exhibit an increased ribosome association concurrent with a decrease in mRNA abundance. (A) Confocal image of a WT control and (B) pelo ${ }^{1}$ mutant ovariole stained with 1B1 (magenta) and Vasa (green) and indicating egg chambers that fail to grow (yellow dashed line) and subsequently die (arrow). (C) Quantification of oogenesis defect phenotypes observed in pelo ${ }^{1}$ mutants $(n=113, p<0.001$, Chi Square Analysis). (D) Venn diagram illustrating overlap of $81 \%$ of Tst-regulated RNAs that are $>2$ fold upregulated upon loss of pelo ( $p<1.49 \times 10^{-151}$, Hypergeometric Test). (E) Biplot of poly(A) mRNA Input $\log _{2}$ TPM versus polysome associated mRNA $\log _{2}$ TPM from adult WT ovaries highlighting Tst-regulated RNAs (magenta) showing low RNA abundance. (F) Biplot of poly(A) mRNA Input $\log _{2}$ TPM versus polysome associated mRNA $\log _{2}$ TPM from undifferentiated germ cells highlighting Tst-regulated RNAs (magenta) indicating both an increased RNA abundance 
810 and ribosome association compared to Adult WT. (G) Biplot of poly(A) mRNA input $\log _{2}$ TPM 811 versus polysome associated mRNA $\log _{2}$ TPM from differentiating cysts highlighting Tst-regulated 812 RNAs (magenta) indicating both an increased RNA abundance and ribosome association 813 compared to Adult WT. (H) Biplot of poly(A) mRNA input $\log _{2}$ TPM versus polysome associated 814 mRNA $\log _{2}$ TPM in germline tst RNAi ovaries highlighting Tst-regulated RNAs (magenta) 815 indicating both an increased RNA abundance and ribosome association compared to Adult WT. 816 (I) Biplot of poly(A) mRNA input $\log _{2}$ TPM versus polysome associated mRNA $\log _{2}$ TPM in pelo ${ }^{1}$ 817 ovaries highlighting Tst-regulated RNAs (magenta) indicating both an increased RNA abundance 818 and ribosome association compared to adult WT. Scale bars are 10 $\mu \mathrm{m}$. (J) Quantification of 819 normalized $\log _{2}$ polysome/input mRNA of Tst-regulated RNAs in CB, cyst, adult, tst RNAi and pelo 820 samples showing increased ribosome association during the transition from CB to cyst to adult. 821 Ribosome association is comparable for cyst and tst RNAi and adult and pelo in which RNA 822 degradation is not occurring. 

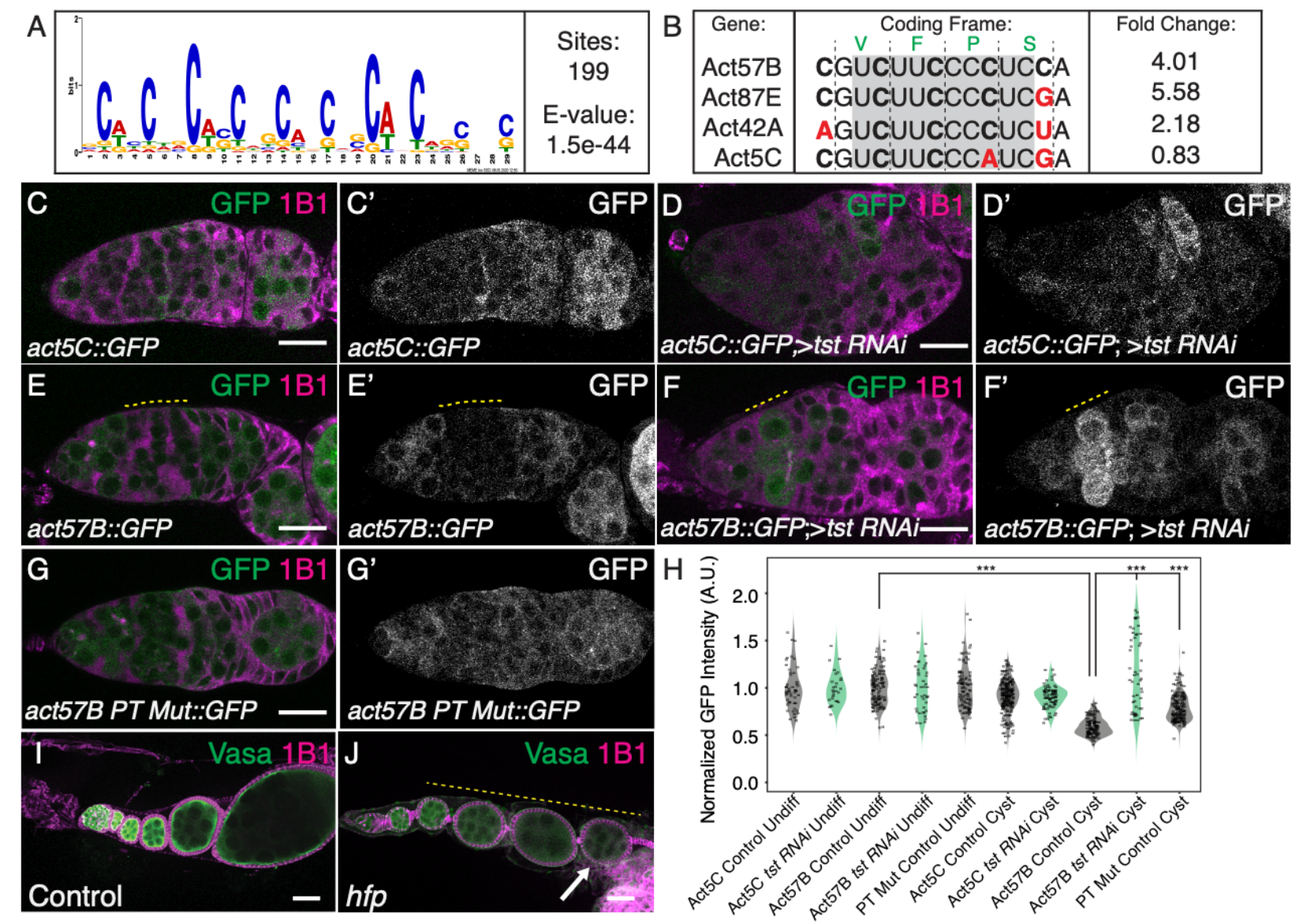

$\mathrm{K}$

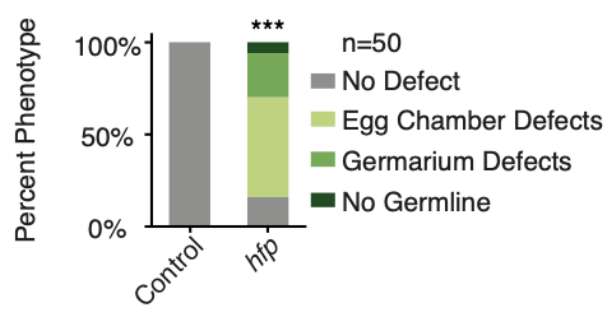

$\mathrm{L}$
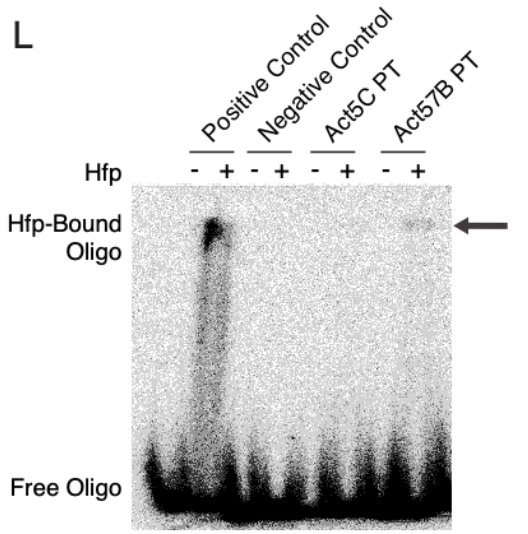

N

$\mathrm{N}$ RNAs

Before Differentiation:
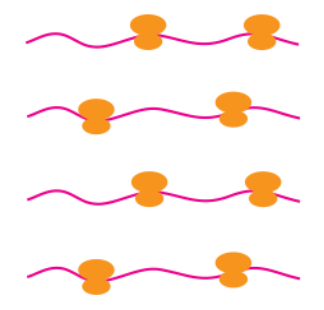

Tst regulated
Mfp

Regulated RNAs

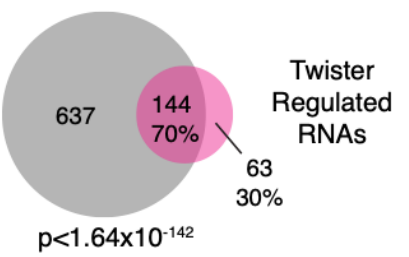

$\mathrm{p}<1.64 \times 10^{-142}$

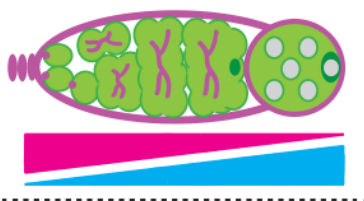

Oocyte Specification

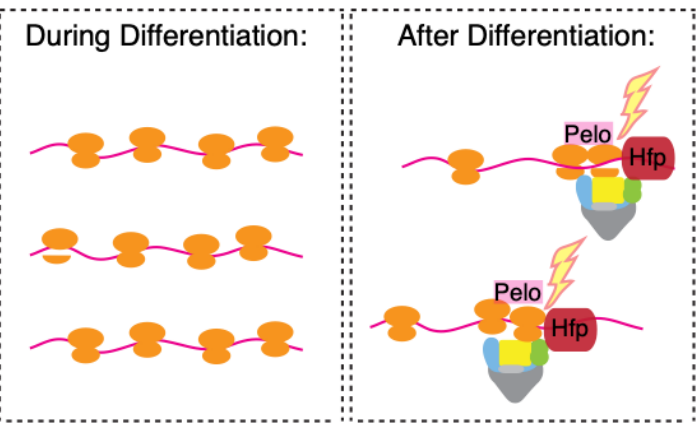



that can be bound by Half Pint. (A) MEME logo of the polypyrimidine-rich motif enriched in the CDS of Tst-regulated RNAs. (B) CDS alignment of polypyrimidine-rich tracts (PTs) found in the Tst-regulated RNAs act57B, act87E, act42A, the non-target paralog act5C and their respective fold changes upon loss of tst. Black vertical lines indicate coding frame with amino acid symbols above. Probe for EMSA experiments boxed in gray and purine transitions in act5C highlighted in red. (C-C') Germarium showing the expression of germline actin5C::GFP fusion reporter (green, grayscale) in control and (D-D') tst RNAi stained with 1B1 (magenta) that does not change in the cysts. (E-E') Germarium showing the expression of germ line actin57B::GFP fusion reporter (green, grayscale) and stained with 1B1 (magenta) showing a decrease in actin57B::GFP expression level in cysts (yellow dashed line) and in (F-F') tst RNAi showing higher actin57B::GFP expression level in cysts (yellow dashed line) compared to control. (G-G') Germarium showing the expression of germ line actin57B PT Mutant::GFP fusion reporter (green, grayscale) in WT stained with 1B1 (magenta) showing consistent actin57B PT Mutant::GFP in both undifferentiated and cyst stages. (H) A.U. quantification of reporter GFP intensity in undifferentiated cells and cyst stages in WT indicating significantly lower actin57B::GFP expression in WT cysts compared to undifferentiated cells. Expression of actin57B::GFP is significantly higher in tst RNAi cysts compared to WT control cysts. Expression of actin57B PT Mutant::GFP is significantly higher than actin57B::GFP in WT control cysts. (actin57B::GFP Control undifferentiated cells $n=110$, actin57B::GFP Control Cyst $\mathrm{n}=116, \mathrm{p}<0.0001$, actin57B::GFP tst RNAi Cyst $\mathrm{n}=53, \mathrm{p}<0.0001$, actin57B PT Mutant::GFP Cyst $n=158, p<0.0001$ Student's t-Test). (I) Confocal image of Control and (J) hfp mutant ovariole stained with 1B1 (magenta) and Vasa (green) showing egg chambers that do not grow in size (yellow dashed line) and dying egg chamber (arrow). (K) Quantification of $h f p$ oogenesis defect phenotypes compared to control ( $n=50, p<0.001$, Chi Square Analysis). (L) EMSA of recombinant Hfp N-terminal RRMs shows that Hfp RRMs bind the Drosophila consensus polypyrimidine-rich sequence (Positive Control) and act57B PT sequence (from B) but not the random scramble sequence (Negative Control), or the act5C PT sequence. (M) Venn diagram illustrating overlap of $70 \%$ of Tst-regulated RNAs upregulated upon loss of $h f p$ $\left(p<1.64 \times 10^{-142}\right.$, Hypergeometric Test). Scale bars are $10 \mu \mathrm{m}$. (N) In undifferentiated cells Tstregulated RNAs are highly expressed, yet lowly associated with ribosomes, and required for early oogenesis. During differentiation, translation of Tst-regulated RNAs increases. After differentiation, during oocyte specification, Hfp protein binds in the CDS of Tst-regulated RNAs leading to targeting by Pelo, and the Ski complex. 
A

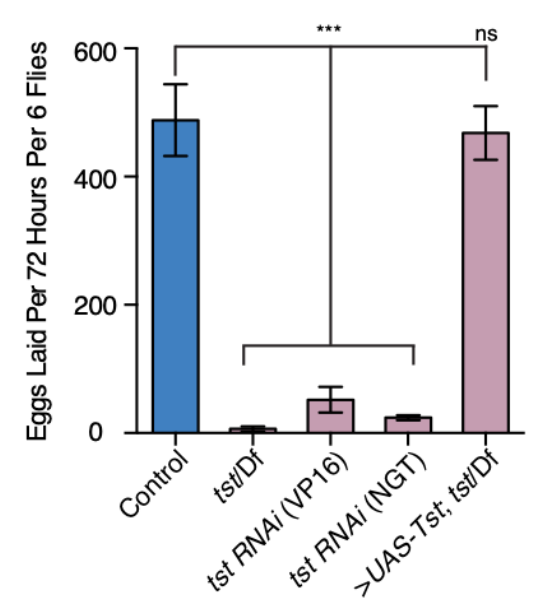

$\mathrm{H}$

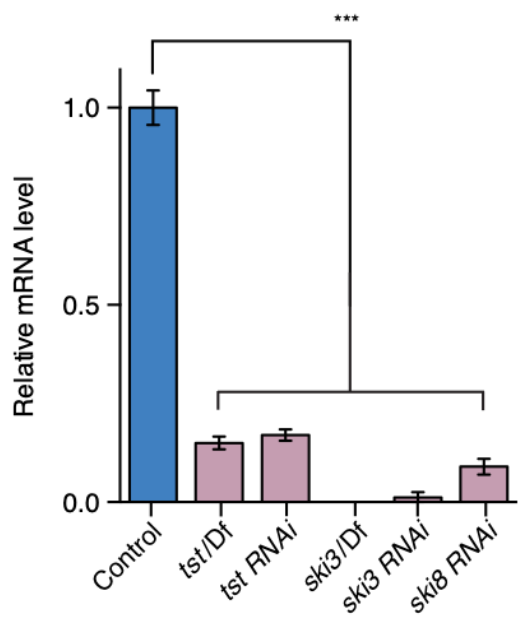

B
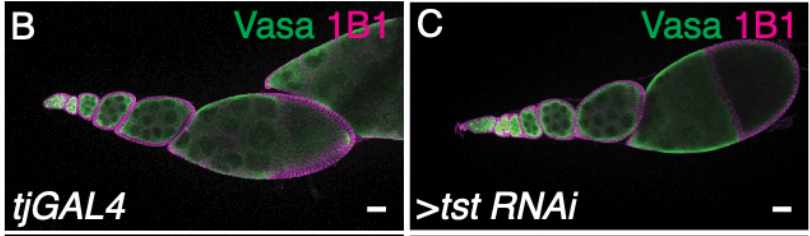

D

Casp3 1B1
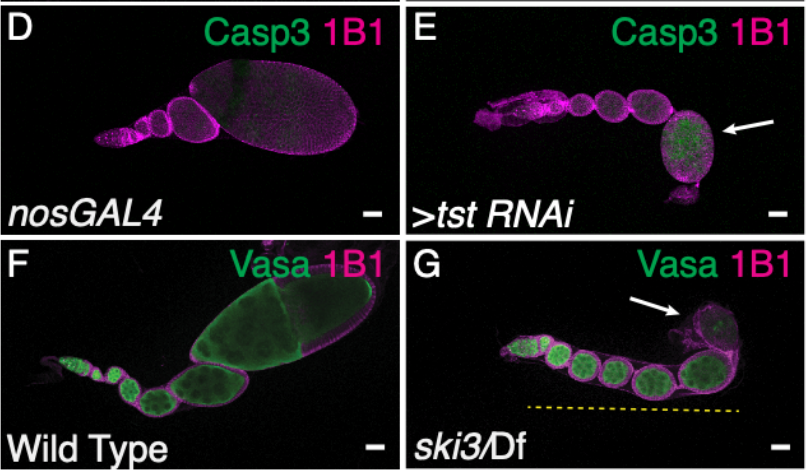

859
Figure S1. Ski Complex components are required for successful oogenesis. (A) Egg laying test assaying the fertility of several Ski complex mutants and germline RNAi knockdown females indicating a loss of fertility compared to control (Control vs tst/Df $\mathrm{n}=3, \mathrm{p}<0.001$, Control vs tst $R N A i$ (VP16), $\mathrm{n}=3, \mathrm{p}<0.001$, Control vs tst RNAi (NGT), $\mathrm{n}=3, \mathrm{p}<0.001$, Control vs UAS-Tst;tst/Df, $\mathrm{n}=3$, not significant (ns) p>0.05, Error bars are standard deviation (SD), Student's t-Test). (B) tjGAL4 driver control and (C) tst RNAi ovarioles stained with Vasa (green) and 1B1 (magenta) exhibiting ovarioles that grow in size and generate later stages. (D) nosGAL4 driver control and (E) tst RNAi ovarioles stained with cleaved Caspase 3 (green) and 1B1 (magenta) indicating dying egg chamber (arrow). (F) WT control and (G) ski3 mutant ovarioles stained with Vasa (green) and 1B1 (magenta) indicating egg chambers that do not grow in size (yellow dashed line) and dying egg chambers (arrow). (H) qRT-PCR assaying the levels of tst, ski3 and ski8 in their respective mutant background or germline RNAi normalized to control levels and indicating successful knockdown (tst Control vs tst/Df $\mathrm{n}=3, \mathrm{p}<0.001$, tst Control vs tst RNAi $\mathrm{n}=3, \mathrm{p}<0.001$, ski3 Control vs ski3/Df $\mathrm{n}=3, \mathrm{p}<0.001$, ski3 Control vs ski3 RNAi $\mathrm{n}=3, \mathrm{p}<0.001$, ski8 Control vs ski8 RNAi $\mathrm{n}=3, \mathrm{p}<0.001$, Error bars are SEM, Student's t-Test). Scale bars are $10 \mu \mathrm{m}$. 

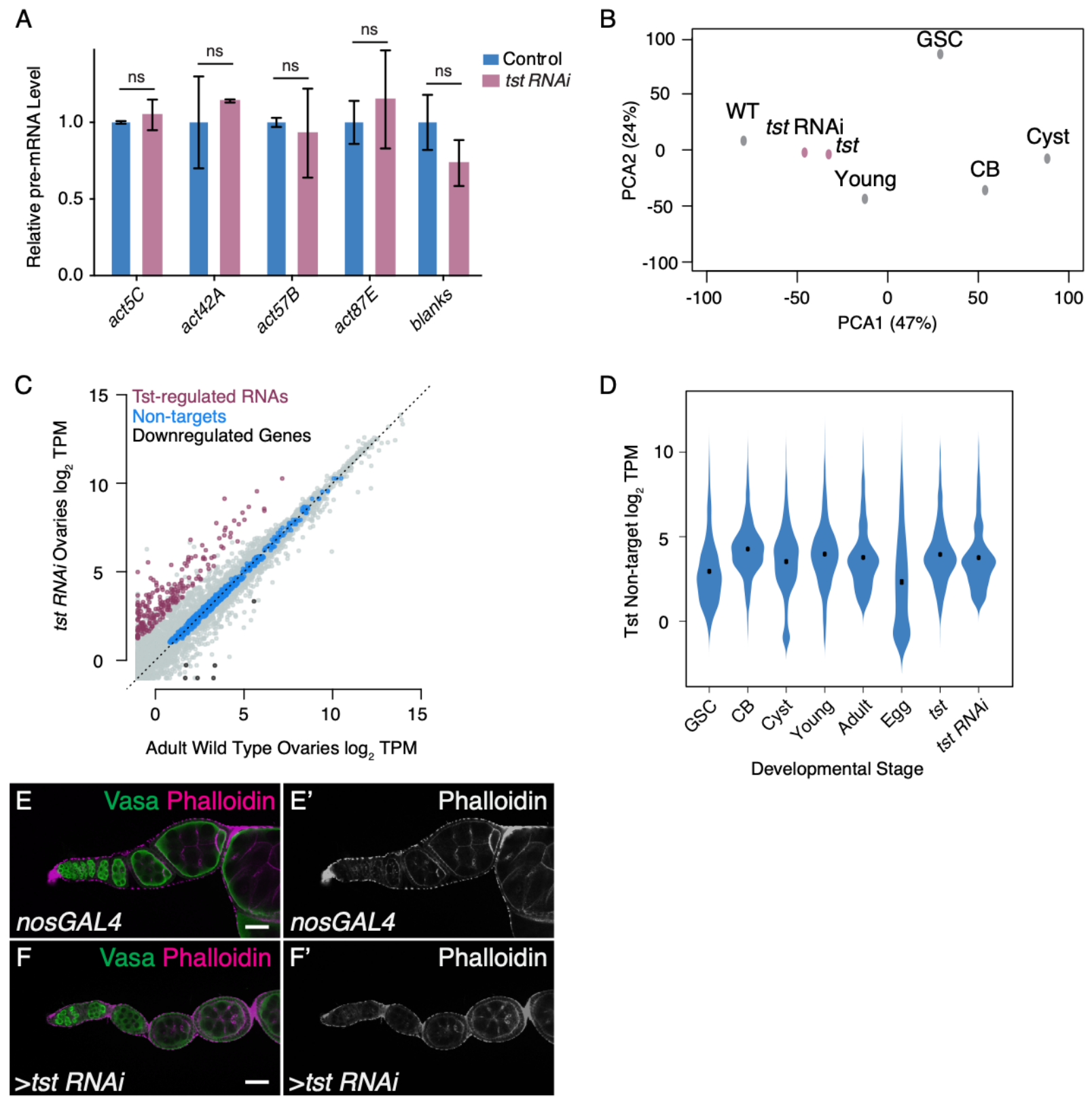

875
Figure S2. Tst post-transcriptionally regulates its target RNAs. (A) qRT-PCR assaying the pre-mRNA levels of several Tst-regulated target genes, including blanks, act42A, act57B, act87E and the non-target act5C in Control and germline tst RNAi normalized to control levels and indicating similar pre-mRNA levels in both conditions (act5C pre-mRNA Control level vs tst RNA $i$ $\mathrm{n}=2$, ns, $\mathrm{p}>0.05$, act42A pre-mRNA Control level vs tst RNAi $\mathrm{n}=2$, ns, $\mathrm{p}>0.05$, act57B pre-mRNA Control level vs tst RNAi $\mathrm{n}=3$, ns, $\mathrm{p}>0.05$, act87E pre-mRNA Control level vs tst RNAi $\mathrm{n}=2$, ns, $\mathrm{p}>0.05$, blanks pre-mRNA Control level vs tst RNAi $\mathrm{n}=3, \mathrm{~ns}, \mathrm{p}>0.05$, Error bars are SEM, Student's t-Test). (B) Principal Component Analysis (PCA) comparing several ovary RNA-seq data sets including, adult (WT), tst RNAi, tst genomic mutant (tst), young WT (Young), germline stem cell enriched (GSC), undifferentiated cystoblast enriched (CB), and differentiating cyst enriched (Cyst). This indicates that the tst mutant and tst RNAi samples are similar to Adult WT. 
(C) Biplot of RNA-Seq data from Adult WT and tst germ line RNAi knockdown ovaries in $\log _{2}$ Transcripts Per Million (TPM) highlighting upregulated Tst-regulated RNAs (magenta), non-target RNAs (blue), and RNAs concordantly downregulated in both tst RNAi and tst genomic mutant ovaries (black). (D) Violin plot assaying the expression of non-target genes that do not substantially change in several RNA-seq data sets including Germline Stem Cell enriched (GSC), undifferentiated Cystoblast enriched (CB), and differentiating cyst enriched (Cyst), young WT (Young), adult (WT), unfertilized eggs (Egg), tst genomic mutant (tst), and germline tst RNAi (tst $R N A i)$. (E-E') nosGAL4 driver control and (F-F') tst RNAi ovarioles stained with Vasa (green) and Phalloidin (magenta and grayscale) indicating similar levels of phalloidin staining. Scale bars are

A

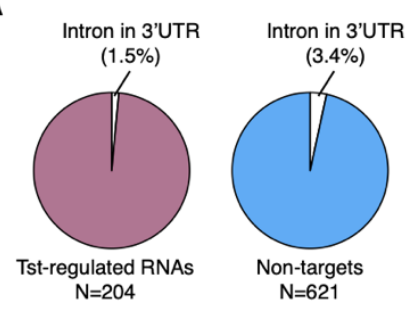

B

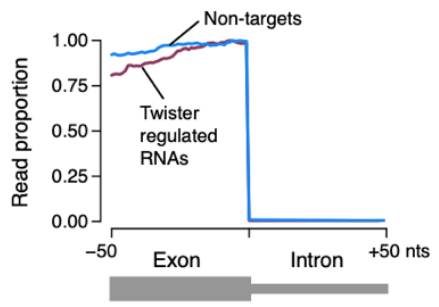

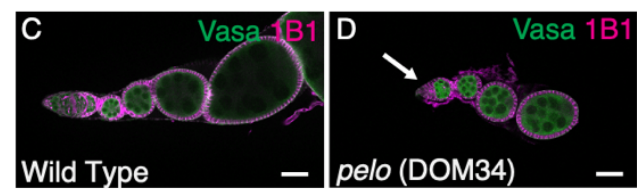

E

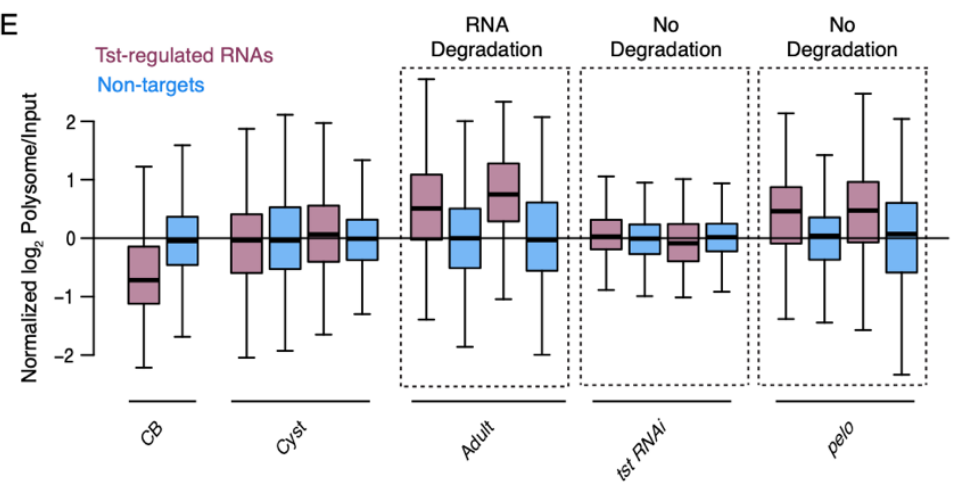

900

901

902

903

904

905

906

907

908

909

910
Figure S3. Tst-regulated RNAs exhibit hallmarks of NGD, but not NMD. (A) Pie graphs showing the percent of Tst-regulated RNAs (magenta) and non-target mRNAs containing an intron in their 3'UTR indicating that a smaller proportion of Tst-regulated RNAs contain an intron in their 3'UTR (1.5\%) compared to non-target RNAs (3.4\%). (B) Metaplots showing the proportion of RNA-Seq coverage mapping to exon-intron boundaries for both Tst-regulated targets (magenta) and non-targets (blue) indicating that both Tst-regulated RNAs and non-target RNAs are spliced correctly. (C) WT control and (D) pelo mutant ovarioles stained with Vasa (green) and

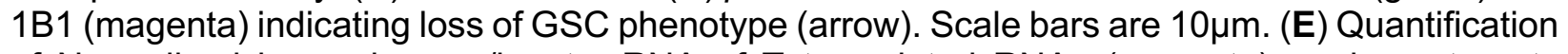
of Normalized $\log _{2}$ polysome/input mRNA of Tst-regulated RNAs (magenta), and non-targets 
bioRxiv preprint doi: https://doi.org/10.1101/2020.06.30.179986; this version posted July 2, 2020. The copyright holder for this preprint (which was not certified by peer review) is the author/funder, who has granted bioRxiv a license to display the preprint in perpetuity. It is made available under aCC-BY-NC-ND 4.0 International license.

911 (blue) in CB, cyst, adult, tst RNAi and pelo samples indicating that ribosome association of Tst912 regulated RNAs is dynamic during development, but not for non-target RNAs.

913

914 
A

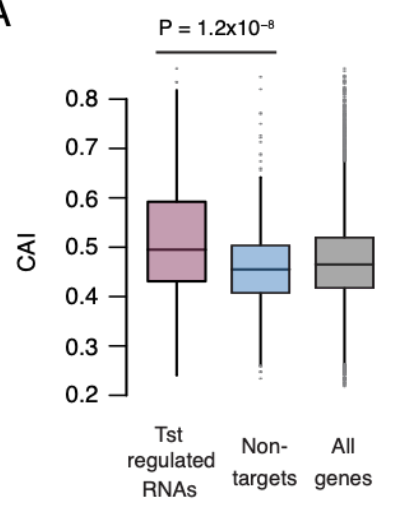

B

\begin{tabular}{|l|r|}
\hline Gene & \multicolumn{1}{|l|}{ CAI } \\
\hline Act5C & 0.79 \\
\hline Act42A & 0.63 \\
\hline Act57B & 0.80 \\
\hline Act87E & 0.78 \\
\hline
\end{tabular}
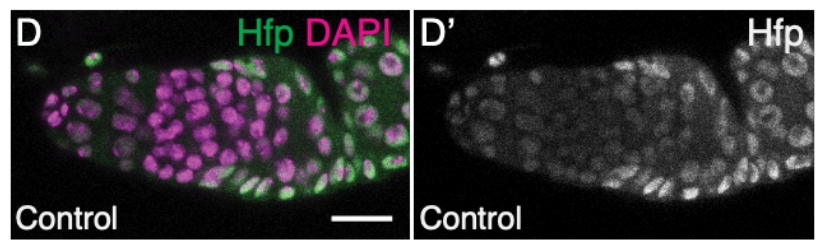

F

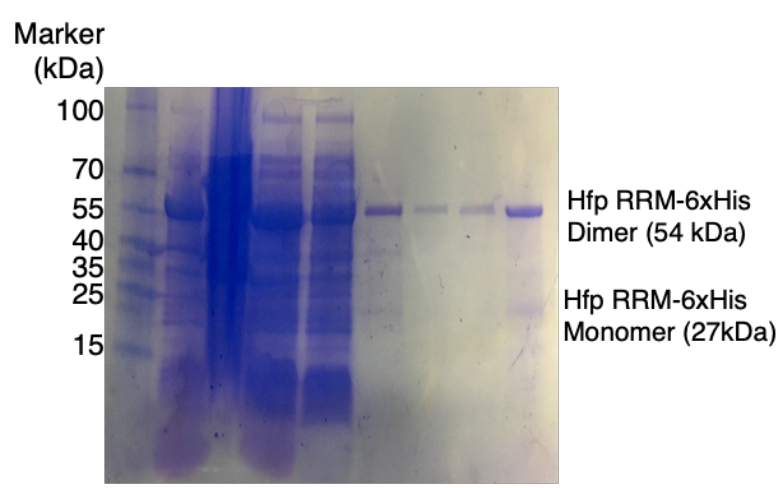

C

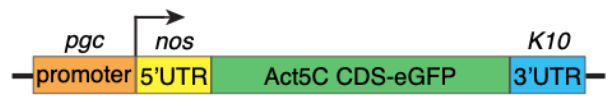

$C^{\prime}$

\begin{tabular}{|c|c|}
\hline$p g c$ & nos \\
\hline
\end{tabular}

C"

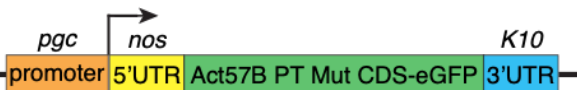

E input Cytoplasm Nucleus

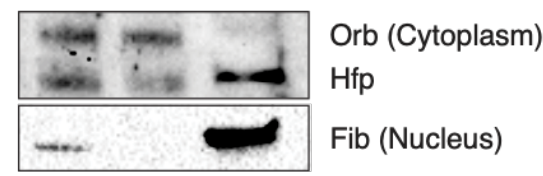

G

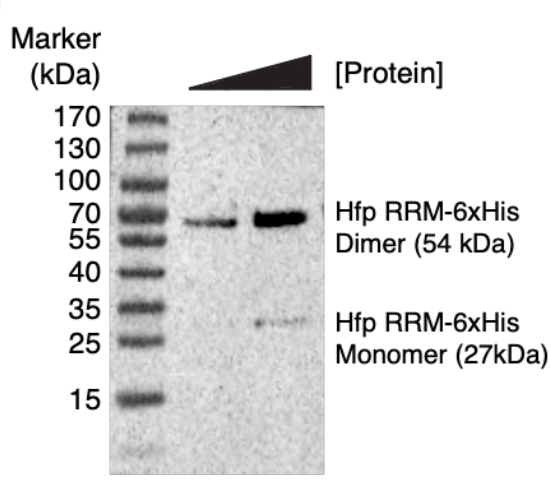

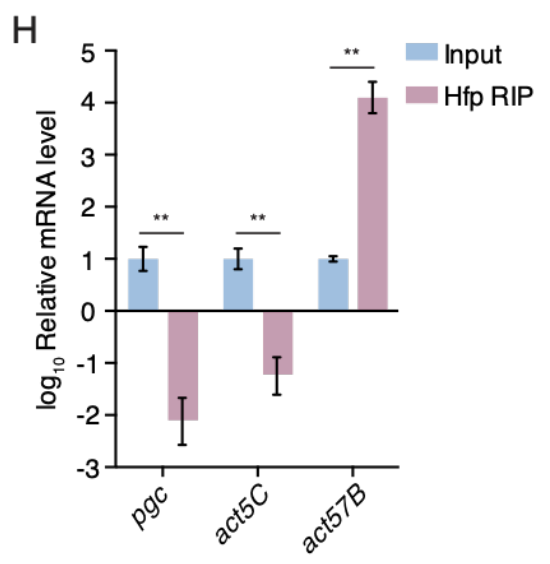

I
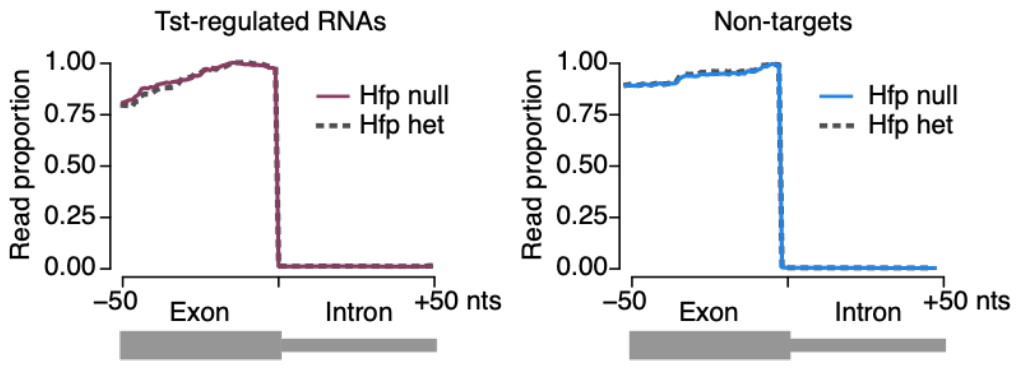

915

916

917

918

Figure S4. Tst-regulated RNAs are bound by Hfp and do not exhibit suboptimal codon usage. (A) Codon Adaptation Index (CAI) comparison for Tst-regulated RNAs (magenta) versus 
non-targets (blue) indicating a higher CAl for Tst-regulated RNAs (Wilcoxon rank sum test); and all genes (gray). (B) Table of the Actin paralog genes and their respective CAl values indicating that they are all very similar. (C) Schematic of the act5C::GFP, (C') act57B::GFP and (C') act57B PT Mutant::GFP reporters under the control of a germline promoter (pgc) and 5'UTR (nos), and a neutral 3'UTR (K10). (D-D') Control germaria stained for Hfp (green and grayscale) and DAPI (magenta) indicating cytoplasmic Hfp expression during early oogenesis. (E) Subcellular fractionation Western blot analysis of input, cytoplasm and nucleus for Hfp, Orb and Fibrillarin indicating that $\mathrm{Hfp}$ is present in both the nucleus and cytoplasmic fractions. (F) SDS-PAGE of a protein marker (lane 1), bacterial supernatant (lane 2), pellet (lane 3), washes (lanes 4-6), and elutions (lanes 7-9) of the Hfp-RRM protein purification process. (G) Western blot analysis of the Hfp-RRM 6X-His Tag showing both monomer and dimer bands. (H) Hfp-HA RIP and qRT-PCR analyses indicating a de-enrichment of non-target $p g c$ and act5C levels and an enrichment of target act57B levels in Hfp RIP samples compared to input ( $p g c$ Input vs Hfp-IP $n=2, p<0.008$, act5C Input vs Hfp-IP $n=2, p<0.005$, act57B Input vs Hfp-IP $n=2, p<0.005$, Error bars are standard error of the mean (SEM), Student's t-Test). (I) Metaplot of the proportion of RNA-seq coverage mapping to exon-intron boundaries in hfp mutant and control (heterozygous) RNA-seq data sets for both Tst-regulated RNAs (magenta) and non-targets (blue) indicating correct splicing in both samples. Scale bars are $10 \mu \mathrm{m}$.

A
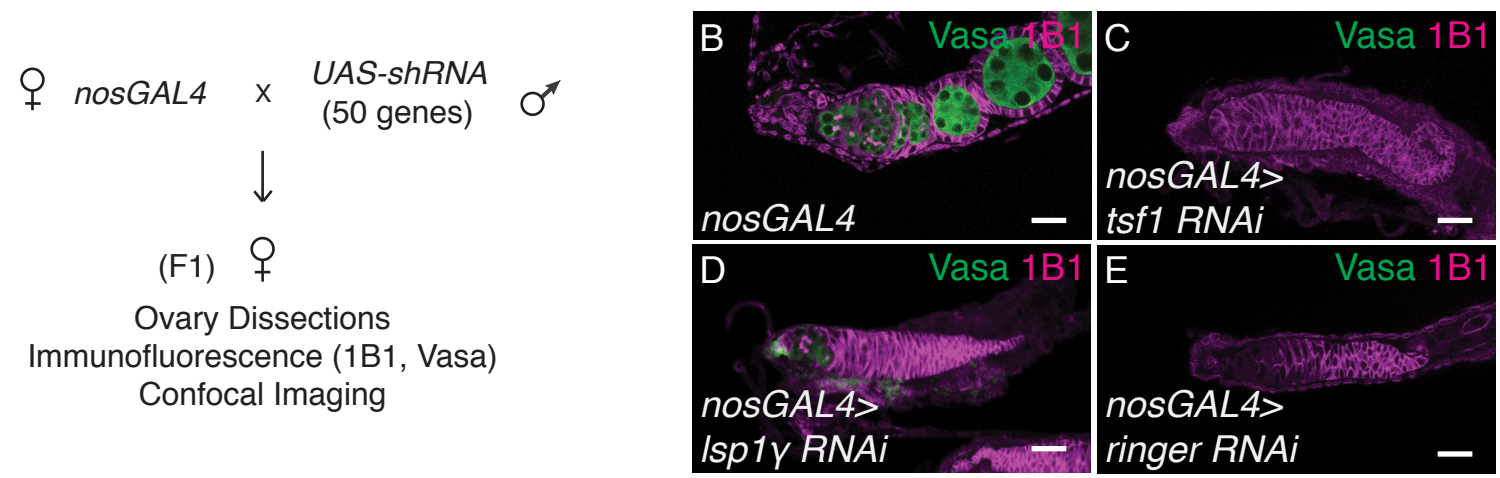

Figure S5. A subset of Tst-regulated RNAs are required for oogenesis. (A) Schematic of the germ line RNAi knockdown screen of Tst-regulated genes. 50 Tst-regulated genes were individually depleted by RNAi in the germline by the UAS-GAL4 system and nosGAL4 driver. F1 ovaries were dissected and phenotypes were assessed by $1 \mathrm{~B} 1$ and Vasa staining and confocal imaging. (B) nosGAL4 driver control, (C) tsf1 RNAi, (D) Isp1y RNAi, and (E) ringer RNAi stained with Vasa (green) and 1B1 (magenta) each exhibiting a complete loss of germ line. Scale bars are $10 \mu \mathrm{m}$. 


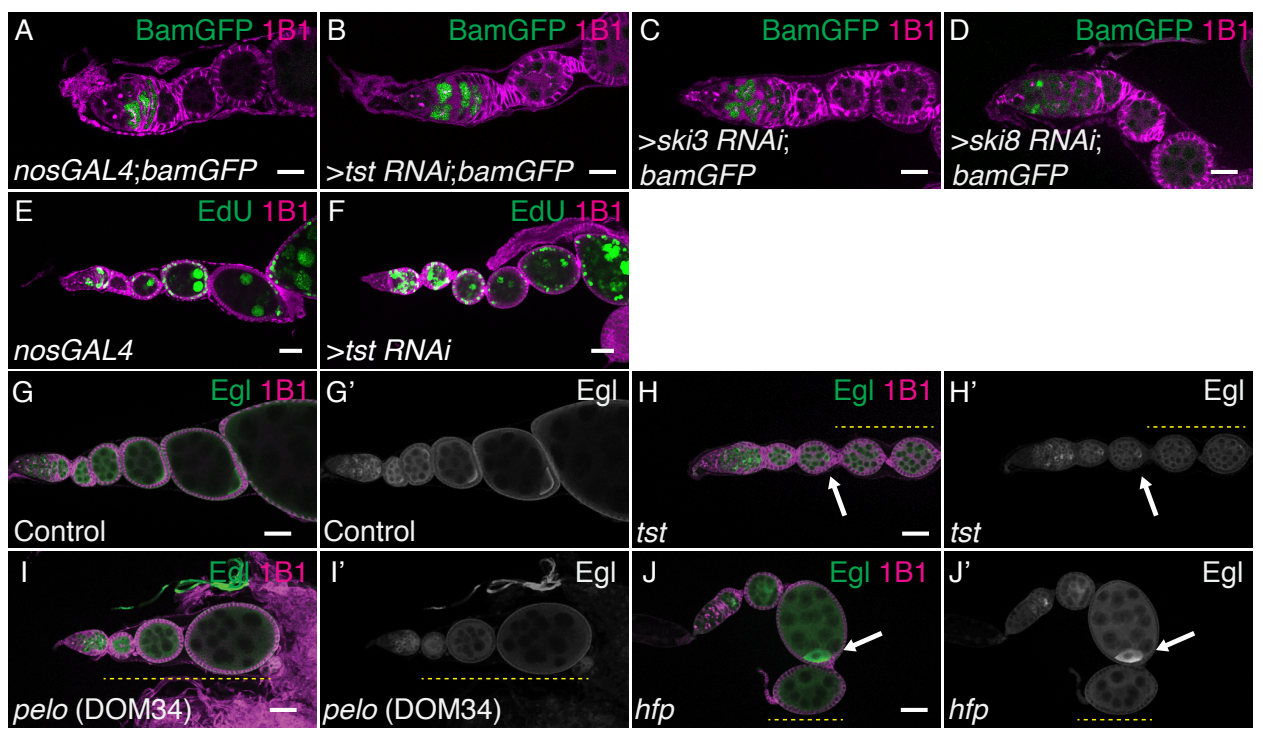

Figure S6. Tst is required for maintaining oocyte fate during oogenesis. (A) nosGAL4; bamGFP driver control ovariole, (B) >tst RNAi;bamGFP, (C) >ski3 RNAi; bamGFP, and (D) >ski8 RNAi;bamGFP ovarioles stained with 1B1 (magenta) and GFP (green) indicating appropriate bamGFP expression for all samples in the differentiating cells. (E) nosGAL4 driver control and (F) >tst RNAi ovarioles stained for EdU (green) and $1 \mathrm{~B} 1$ (magenta) indicating that endocycling is occurring properly. Scale bars are 10 $\mu \mathrm{m}$. (G-G') WT control, and (H-H') tst RNAi ovarioles stained with 1B1 (magenta) and Egl (green and grayscale) showing initial localization of Egl (arrow) and subsequent loss of Egl accumulation in tst RNAi ovarioles (yellow dashed line). (I-I') pelo and (J-J') hfp mutant ovarioles stained with 1B1 (magenta) and Egl (green and grayscale) showing initial Egl localization (arrow) and subsequent loss of Egl accumulation (yellow

960 dashed lines). 